\title{
Especies vegetales con uso potencial en la remediación de zonas contaminadas en México
}

\author{
Plant species with potential use in the remediation \\ of polluted areas in Mexico
}

\author{
José G. Chan-Quijano ${ }^{1,2, \star}$, Manuel J. Cach-Pérez ${ }^{3}$ y Marilú López-Mejía ${ }^{4}$
}

\begin{abstract}
Recibido: 07 enero 2021 | Aceptado: 27 mayo 2021 | Publicado en línea: 30 junio 2021 Citación: Chan-Quijano, JG; Cach-Pérez, MJ; López-Mejía, M. 2021. Especies vegetales con uso potencial en la remedición de zonas contaminadas en México. Revista Forestal del Perú 36(1): 2246. DOI: http://dx.doi.org/10.21704/rfp.v1i36.1703
\end{abstract}

\begin{abstract}
Resumen
Durante los últimos 60 años, en México la contaminación del suelo y del agua (ya sea superficiales o subterráneas) ha venido en aumento como resultado de la explotación de los recursos naturales a través de diferentes actividades industriales, lo que han provocado serios daños al ambiente natural en casi a la totalidad de los ecosistemas del país. En este sentido, las tecnologías para la remediación de los ambientes contaminados son procesos relativamente sencillos de implementar y desarrollar, sin embargo, requieren del conocimiento de los factores (biológicos, físicos y químicos) involucrados para su correcta aplicación. En este sentido, el objetivo de este trabajo es actualizar la lista botánica de especies promisorias para la remediación de zonas contaminadas en México. Para esto, se realizó una extensa revisión bibliográfica de estudios sobre la remediación de suelos y cuerpos de agua contaminados por hidrocarburos del petróleo y metales pesados. A partir de esto, se elaboró y amplió el listado botánico de especies con uso potencial en procesos remediación. El listado incluyó a 174 especies, de las cuales 126 son nativas y 48 son introducidas, pertenecientes a 53 familias botánicas que crecen y se desarrollan en sitios contaminados. Las familias con mayor
\end{abstract}

\footnotetext{
${ }^{1}$ Universidad Autónoma de Guadalajara, Campus Tabasco. Prol. Paseo Usumacinta km 3.5, Fracc. El Country, C.P. 86039, Villahermosa, Tabasco, México.

${ }^{2}$ El Colegio de la Frontera Sur. Km 15.5 carretera a Reforma, RA El Guineo 2a. Sección, C.P. 86280, Villahermosa, Tabasco, México.

${ }^{3}$ CONACYT - Departamento de Agricultura, Sociedad y Ambiente, El Colegio de la Frontera Sur. Km 15.5 carretera a Reforma, RA El Guineo 2a. Sección, C.P. 86280, Villahermosa, Tabasco, México.

${ }^{4}$ Laboratorio de Biología Evolutiva y Genética de Poblaciones. Universidad de Quintana Roo. Av. Andrés Quintana Roo, calle 11, con calle 110 sur s/n, frente a la Col. San Gervasio, C.P. 77642, Cozumel, Q. Roo, México.

* Autor de Correspondencia: jose.chan@uagtabasco.edu.mx
} 
número de especies en estos ambientes fueron Poaceae (33), Fabaceae (31), Asteraceae (9), Cyperaceae (9) y Solanaceae (7). A partir de este listado, se pueden aplicar estudios sobre la capacidad de restauración de zonas contaminadas y su remediación con distintas especies vegetales e incluso abordar, entre otros factores, las características físicas y químicas del suelo o del agua para los procesos de remediación. De igual forma, es necesario generar información sobre las estrategias que las plantas adoptan para aclimatarse a sitios contaminados, así como el rol que podrían desempeñar en estrategias de fitorremediación (acumulación o transformación de los contaminantes, por ejemplo).

Palabras clave: hidrocarburos, biorremediación, plantas nativas, contaminación, metales pesados

\begin{abstract}
During the last 60 years, in Mexico soil and water contamination (whether superficial or underground) has been increasing due to the exploitation of natural resources by industrial activities, which have caused serious damage to the natural environments, affecting almost all ecosystems in the country. In this sense, the technologies for the remediation of contaminated environments consist in relatively simple processes to implement and develop, however, they require knowledge of the factors (biological, physical, and chemical) involved for their correct application. Therefore, the objective of this work is to update the botanical list of promising species for the remediation of contaminated areas in Mexico. For this, an extensive bibliographic review of studies on the remediation of soils and water bodies contaminated by petroleum hydrocarbons and heavy metals was carried out. Based on this, the botanical list of species with potential use in remediation processes was elaborated and expanded. The list included 174 species, of which 126 are native and 48 are introduced, belonging to 53 botanical families that grow and develop in contaminated sites. The families with the highest number of species in these environments were Poaceae (33), Fabaceae (31), Asteraceae (9), Cyperaceae (9) and Solanaceae (7). From this list, studies can be applied on the restoration capacity of contaminated areas and their remediation with different plant species and even address, among other factors, the physical and chemical characteristics of the soil or water for remediation processes. Similarly, it is necessary to generate information on the strategies that plants adopt to acclimatize to contaminated sites, as well as the role they could play in phytoremediation strategies (accumulation or transformation of pollutants, for example).
\end{abstract}

Key words: hydrocarbons, bioremediation, native plants, pollution, heavy metals

\section{Introducción}

A nivel mundial los derrames de petróleo afectan el suelo y los sistemas acuáticos, e incluso los contaminantes atmosféricos que contienen metales pesados, afectan a la totalidad de los ecosistemas (Fernández-Linares et al. 2006, Rodríguez-Eugenio et al. 2019, Molnár et al. 2020). Por lo tanto, el problema de la contaminación ambiental es, sin duda, uno de los más importantes por resolver.

Es importante entender y articular las distintas dinámicas socioambientales de las problemáticas de la contaminación de los suelos y los sistemas acuáticos, dado que las consecuencias que traen los derrames de petróleo o la contaminación por metales pesados en el suelo y en el agua son la pérdida de calidad derivada de cambios químicos, físicos, biológicos y, en el caso del suelo, reducción en la fertilidad y la capacidad de crecimiento sostenido de algunas plantas. En el agua provoca acidificación que, además de la simple presencia de los contaminantes, los organismos acuáticos no soportan, lo que provoca su mortalidad (Gardi et al. 2014).

La contaminación de los suelos y de los sistemas acuáticos siguen en aumento como resul- 
tado de la explotación de los recursos naturales. Por ello, Velasco-Trejo y Volke-Sepúlveda (2003) y Alarcón y Ferrera-Cerrato (2013) mencionan que al aplicar procesos de remediación se aprovecha a los organismos vivos (plantas, hongos, bacterias, entre otros) para obtener alternativas de recuperación, ya que se extrae, biodegrada o biotransforma a los compuestos orgánicos tóxicos en productos metabólicos menos tóxicos o inocuos. Sin embargo, aún son necesarios estudios sobre la ecofisiología y morfología vegetal de las plantas para conocer sus estrategias de aclimatación y con ello, poder desarrollar estrategias de remediación de los ambientes contaminados (Adki et al. 2013).

Estas alternativas de recuperación y remediación de los sistemas ecológicos contaminados son procesos relativamente sencillos de implementar y desarrollar. Sin embargo, se requieren conocimientos sobre las directrices de remediación y de los ejes de la sostenibilidad para dar un buen manejo de recuperación de los ambientes afectados (Velasco-Trejo y Volke-Sepúlveda 2003, Chan-Quijano et al. 2015).

Muchas especies de plantas son capaces de aclimatarse a la presencia de metales pesados e hidrocarburos del petróleo (HP), a través de diferentes estrategias anatómicas y fisiológicas que les permiten tolerar a los contaminantes en suelo o agua (Muratova et al. 2008, Ochoa-Gaona et al. 2011, Pérez-Hernández et al. 2013). En este sentido, algunas de estas especies tienen valor económico para algunos productores (árboles maderables o como productos para la artesanía), así como para algunas empresas locales, esto, puede utilizarse como estrategia socioeconómica para las poblaciones afectadas por la contaminación de los suelos o sistemas acuáticos y a la par apoyar en la remediación de los sitios.

Además, las especies arbóreas tienen una multifunción en los ecosistemas, como capacidad de acumulación de metales, tolerancia a las condiciones locales del suelo, raíces que llegan a más profundidad a comparación de algunos pastos y arbustos, así como una gran capacidad de captura de carbono (Pérez-Hernández et al. 2013, Burges et al. 2017, Chan-Quijano et al.
2020). Los pastos y arbustos son de rápido crecimiento, rápida propagación y sistema radicular abundante, lo que podría funcionar como alternativas de remediación rápida (Pandey et al. 2014).

Es deseable que todas las especies vegetales a utilizar en la remediación de suelos contaminados sean capaces de iniciar una sucesión ecológica y que las plantas acuáticas logren limpiar los sistemas acuáticos y, que, a su vez, se logre un buen rendimiento para los bienes y servicios a la sociedad. Con esto, surge la necesidad de elaborar un listado botánico de especies con uso potencial en procesos de remediación de zonas contaminadas. Estas especies vegetales tienen capacidades fisiológicas y anatómicas para utilizarlas como alternativa de un manejo-técnico biológico para los planes y procesos de remediación. Si no se toman acciones en las próximas décadas para atender las problemáticas de los suelos y sistemas acuáticos contaminados, estos serán irremediables. Por lo tanto, se retoma la lista de Chan-Quijano et al. (2013) para actualizar las especies vegetales promisorias en la remediación de zonas contaminadas.

\section{Materiales y Métodos}

Se realizó una búsqueda extensiva entre 2017 y 2020 de artículos científicos y de divulgación, así como de libros (digitales e impresos), capítulos de libro, tesis y reportes de investigación en las temáticas de biorremediación, fitorremediación, remediación de suelos y sistemas acuáticos contaminados y fitorremediación con uso de especies vegetales que crezcan, se desarrollen, toleren y degraden hidrocarburos del petróleo y/o bioacumulen metales pesados. La revisión se realizó en la base de datos de la biblioteca digital de la Universidad Autónoma de Guadalajara, Campus Tabasco (http://tabasco.uag.mx/Universidad/Biblioteca), del Sistema de Información Bibliotecario de El Colegio de la Frontera Sur (SIBE; http://bibliotecasibe. ecosur.mx/sibe/) y en el Consorcio Nacional de Recursos de Información Científica y Tecnológica (CONRICYT; https://www.conricyt.

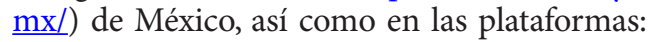


Scopus (https://www.scopus.com/home.uri), Redalyc (https://www.redalyc.org/), Dialnet (https://dialnet.unirioja.es/), Elsevier (https:// www.elsevier.com/es-mx), Jstor (https://www. jstor.org/), Taylor \& Francis (https://www. tandfonline.com/), Springer (https://www. springer.com/la), Scielo (https://www.scielo. org/), Science (https://www.sciencemag.org/) y Nature (https://www.nature.com/). En la búsqueda se introdujeron palabras clave y tesauros como: biorremediación, plantas nativas e introducidas, fitorremediación, plantas acuáticas y remediación de sistemas acuáticos, remediación de suelos contaminados, degradación de contaminantes en suelos con uso de plantas, y remediación en México. Se revisaron 500 documentos publicados entre 1990 y 2020, de los cuales se seleccionaron 97 que mencionaran especies vegetales nativas e introducidas en México (en campo y vivero). A partir de los documentos encontrados se revisó el nombre científico y común (en caso de reportarla) de las especies vegetales utilizadas en procesos de remediación de suelos y sistemas acuáticos contaminados y también se consideró su ambiente caracterizado y contaminante presente. Para la clasificación de especie introducida o nativa, si no fue mencionado en el documento, se utilizó el listado de The Plant List (2013), Herbario virtual de la CONABIO (2019) y Global Plants (2020).

\section{Resultados}

Se registraron 53 familias botánicas con un total de 174 especies, de estas 126 son nativas y 48 introducidas. Las familias más representativas con un mayor número de especies fueron Poaceae (33), Fabaceae (31), Asteraceae (9), Cyperaceae (9) y Solanaceae (7), el resto solo presentó entre una y cuatro especies (Cuadro 1; Figura 1). Estas especies se reportan creciendo y desarrollándose en suelos y sistemas acuáticos contaminados por HP y metales pesados.

Los pastos y arbustos son los más reportados en las investigaciones como especies remediadoras. Son pocas las especies arbóreas y acuáticas reportadas como promisorias en la biorremediación de ambientes contaminados.
En este sentido, las especies reportadas en el listado botánico no representan la totalidad de las especies con uso potencial para la remediación de las zonas contaminadas, ya que constantemente se reportan otras especies que toleran contaminantes.

Además, en la revisión bibliografía se aprecia que a partir del año 2003 hubo un aumento en trabajos de investigación sobre biorremediación y fitorremediación de suelos y sistemas acuáticos, siendo el periodo entre 2013 y 2014 donde se registró el mayor número de publicaciones (Figura 2). De igual forma, la mayoría de los trabajos se publican en forma de artículos científicos y de revisión.

Por otra parte, en el listado se encuentra $\mathrm{Ce}$ drela odorata y las cuatro especies de mangle reportadas para México (Avicennia germinans, Conocarpus erectus, Rhizophora mangle, Laguncularia racemosa), las cuales están en las categorías de protegida y amenazadas, respectivamente, dentro de la Norma Oficial Mexicana NOM-059-SEMARNAT-2010 (SEMARNAT 2010). Por lo anterior, su uso en procesos de remediación debe ser con debido cuidado y con permisos de las autoridades competentes (Comisión Nacional Forestal y Secretaría de Medio Ambiente y Recursos Naturales de México). Su correcto uso, además de ayudar a recuperar ambientes contaminados, puede también contribuir a la recuperación de poblaciones de estas estas especies, contribuyendo de esta forma a su conservación.

El uso potencial de las plantas para la remediación de contaminantes es distinto, por lo que la capacidad de degradación, acumulación o volatilización del contaminante será en diferentes concentraciones. De igual forma, lo que se pretende con este listado es tener un documento en donde se concentren los estudios hasta ahora realizados en México, y pueda servir como antecedentes para la utilización de especies vegetales nativas e introducidas en el país para el uso en la remediación de suelos y sistemas acuáticos contaminados como alternativas en el manejo técnico-biológico para la recuperación de los suelos y cuerpos de agua. 


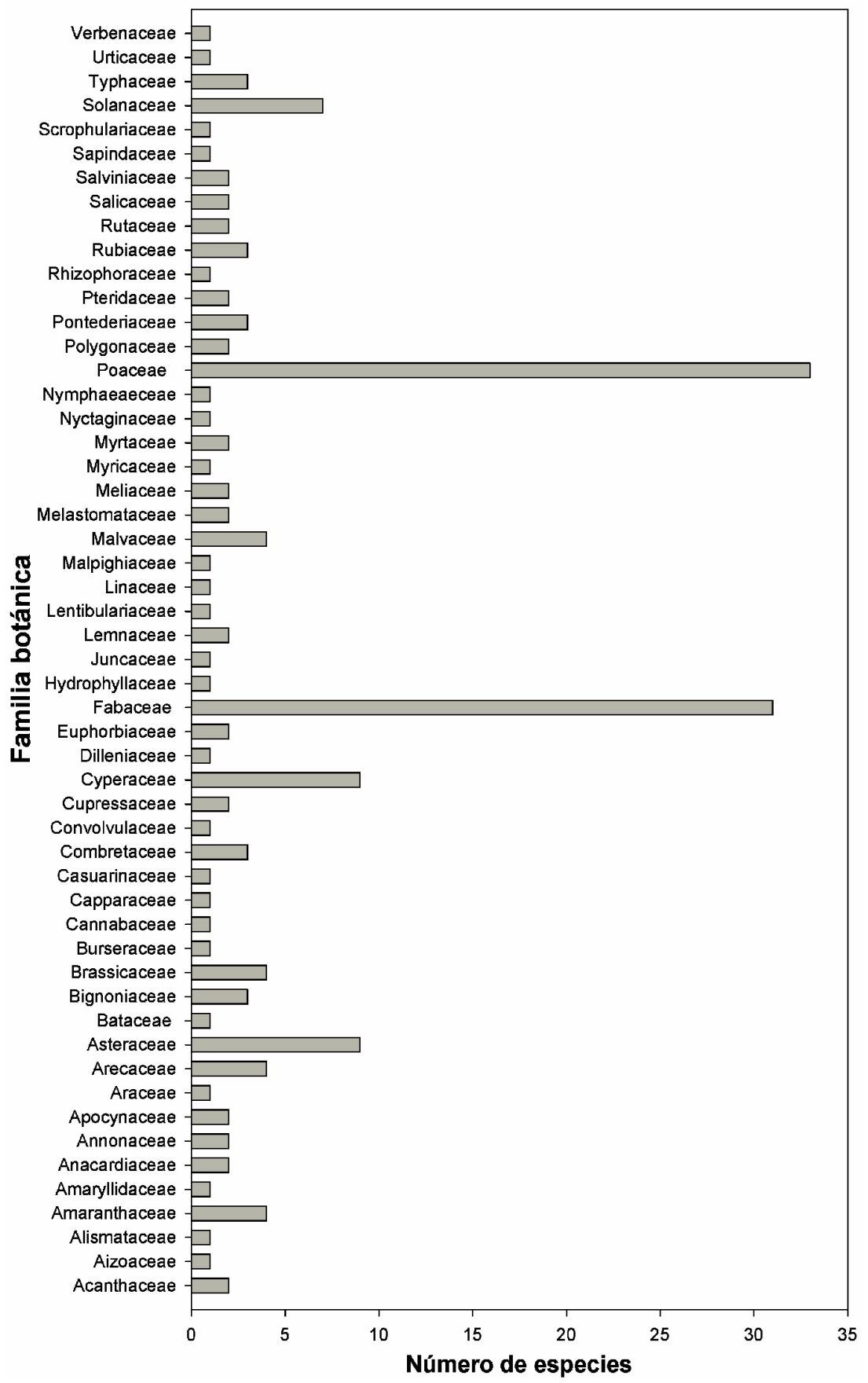

Figura 1. Familia botánica correspondiente a cada especie encontrada. 


\begin{tabular}{|c|c|c|c|c|c|c|c|c|c|c|c|c|c|c|c|c|c|c|}
\hline 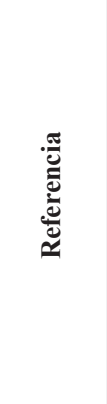 & 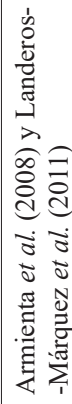 & 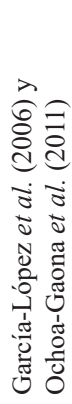 & 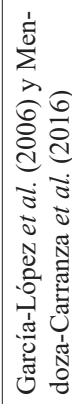 & 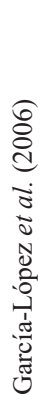 & 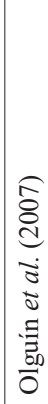 & 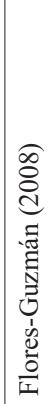 & 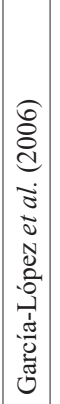 & 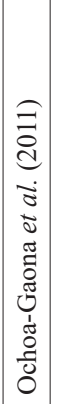 & 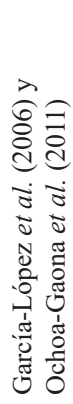 & 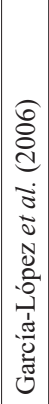 & 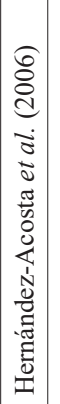 & 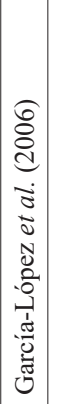 & 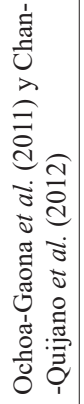 & 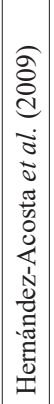 & 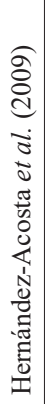 & 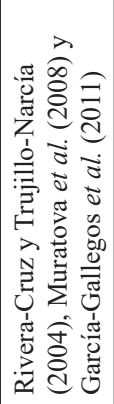 & 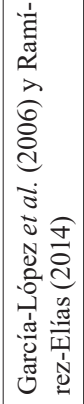 & 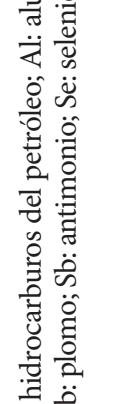 \\
\hline 总 & 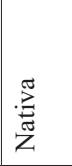 & 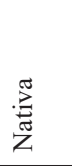 & 胥 & 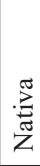 & 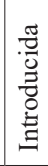 & 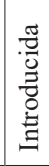 & 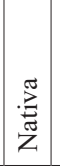 & 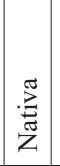 & 胥 & $\begin{array}{l}\stackrel{\widetilde{Z}}{己} \\
\text { Z }\end{array}$ & 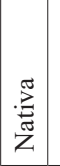 & 胥 & 胥 & $\begin{array}{l}\stackrel{\pi}{己} \\
\stackrel{\pi}{\pi} \\
z\end{array}$ & 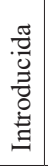 & 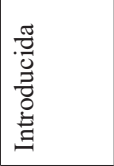 & 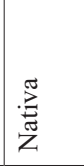 & 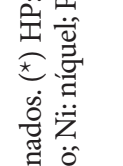 \\
\hline 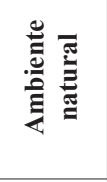 & $\begin{array}{l}\frac{0}{0} \\
\stackrel{\Xi}{*}\end{array}$ & 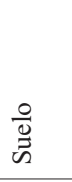 & 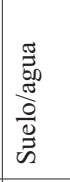 & 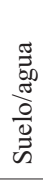 & 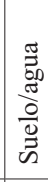 & 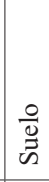 & $\begin{array}{l}0 \\
\stackrel{D}{D} \\
\tilde{n}\end{array}$ & $\begin{array}{l}0 \\
\stackrel{D}{D} \\
\tilde{n}\end{array}$ & $\begin{array}{l}\stackrel{0}{0} \\
\stackrel{\Xi}{0}\end{array}$ & $\begin{array}{l}\frac{0}{0} \\
\stackrel{D}{D}\end{array}$ & $\begin{array}{l}0 \\
\stackrel{D}{D} \\
\tilde{E}\end{array}$ & $\begin{array}{l}0 \\
\stackrel{D}{D} \\
\tilde{n}\end{array}$ & $\begin{array}{l}\frac{0}{0} \\
\ddot{n}\end{array}$ & 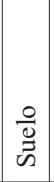 & 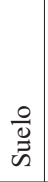 & 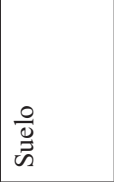 & 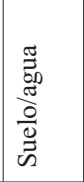 & 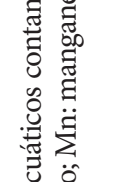 \\
\hline 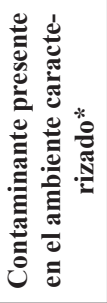 & $\begin{array}{l}0 \\
2 \\
\dot{2}\end{array}$ & 主 & 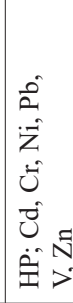 & 主 & 主 & $\hat{2}$ & 主 & 主 & 主 & 至 & 量 & 量 & 量 & $\begin{array}{l}\vec{D} \\
\dot{z} \\
\dot{E} \\
\dot{H} \\
\hat{U}\end{array}$ & 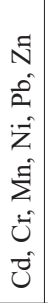 & 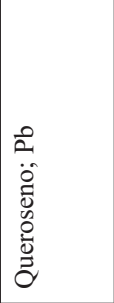 & 主 & 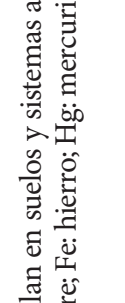 \\
\hline 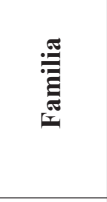 & 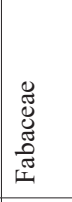 & 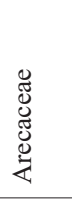 & 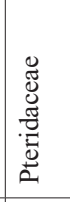 & 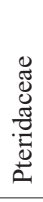 & 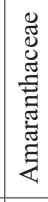 & 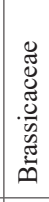 & 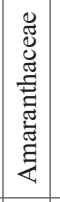 & 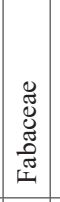 & 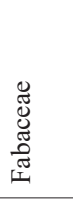 & 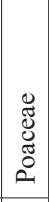 & $\begin{array}{l} \\
\mathscr{J} \\
\mathbb{Z} \\
\tilde{J} \\
2\end{array}$ & 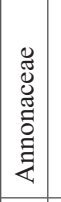 & 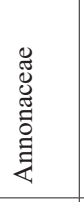 & 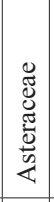 & 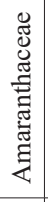 & 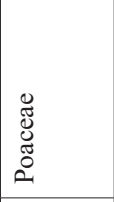 & 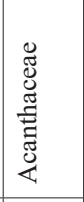 & 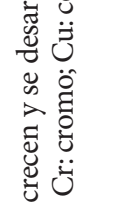 \\
\hline 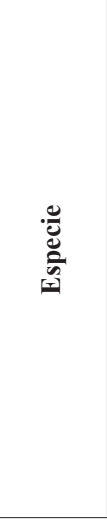 & 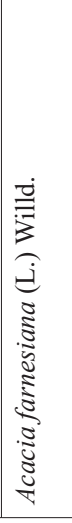 & 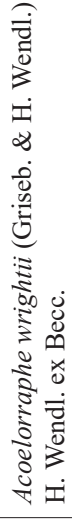 & 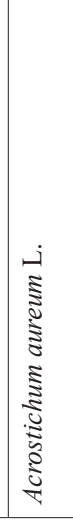 & 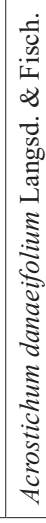 & 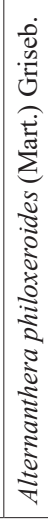 & 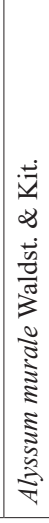 & 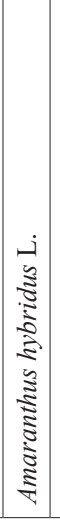 & 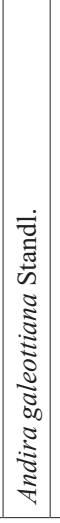 & 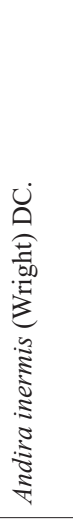 & 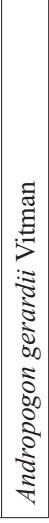 & 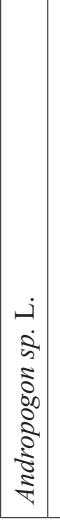 & 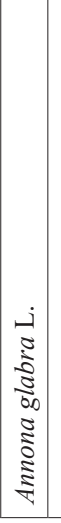 & 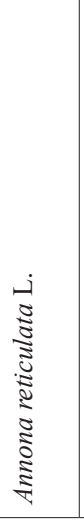 & 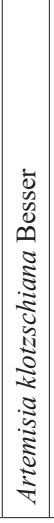 & 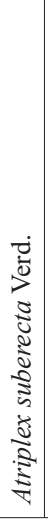 & 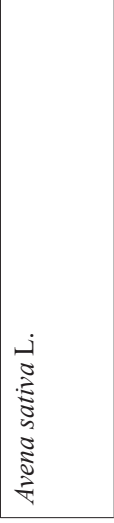 & 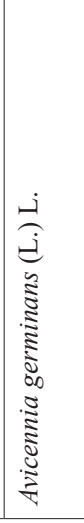 & 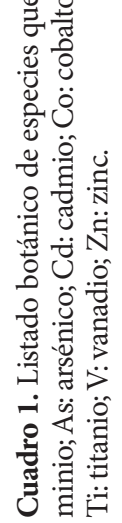 \\
\hline
\end{tabular}




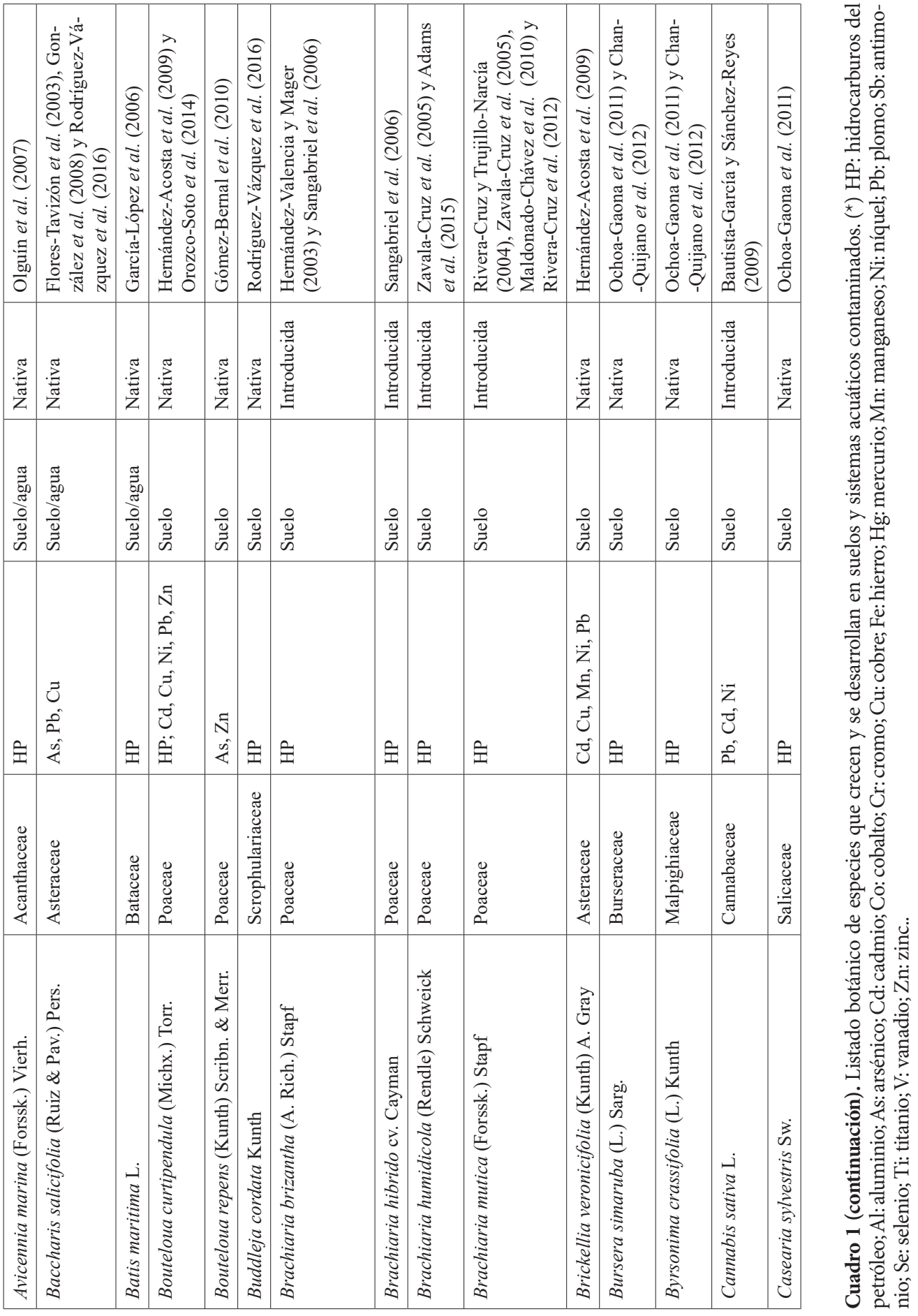




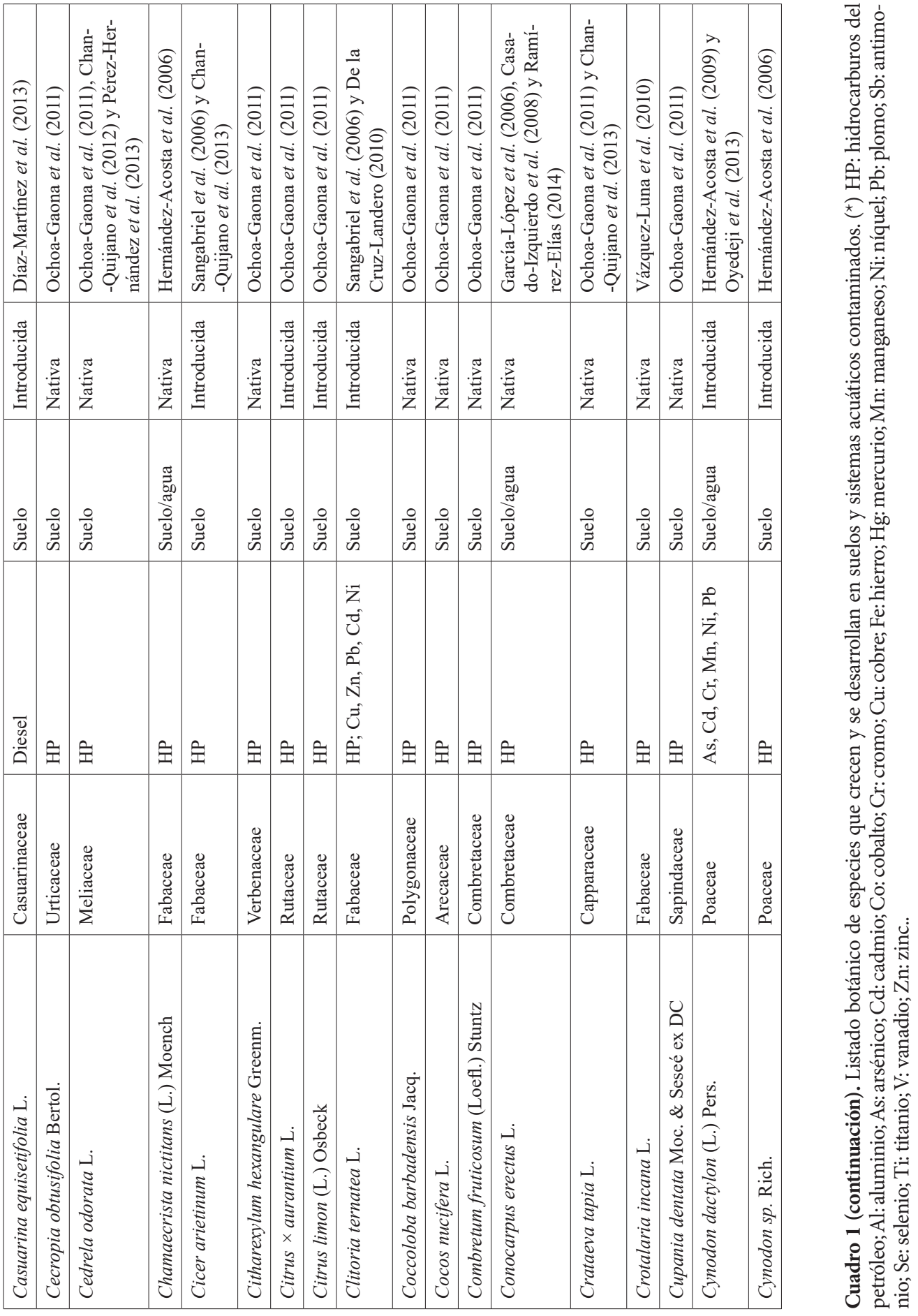


Especies vegetales con uso potencial en la remediación

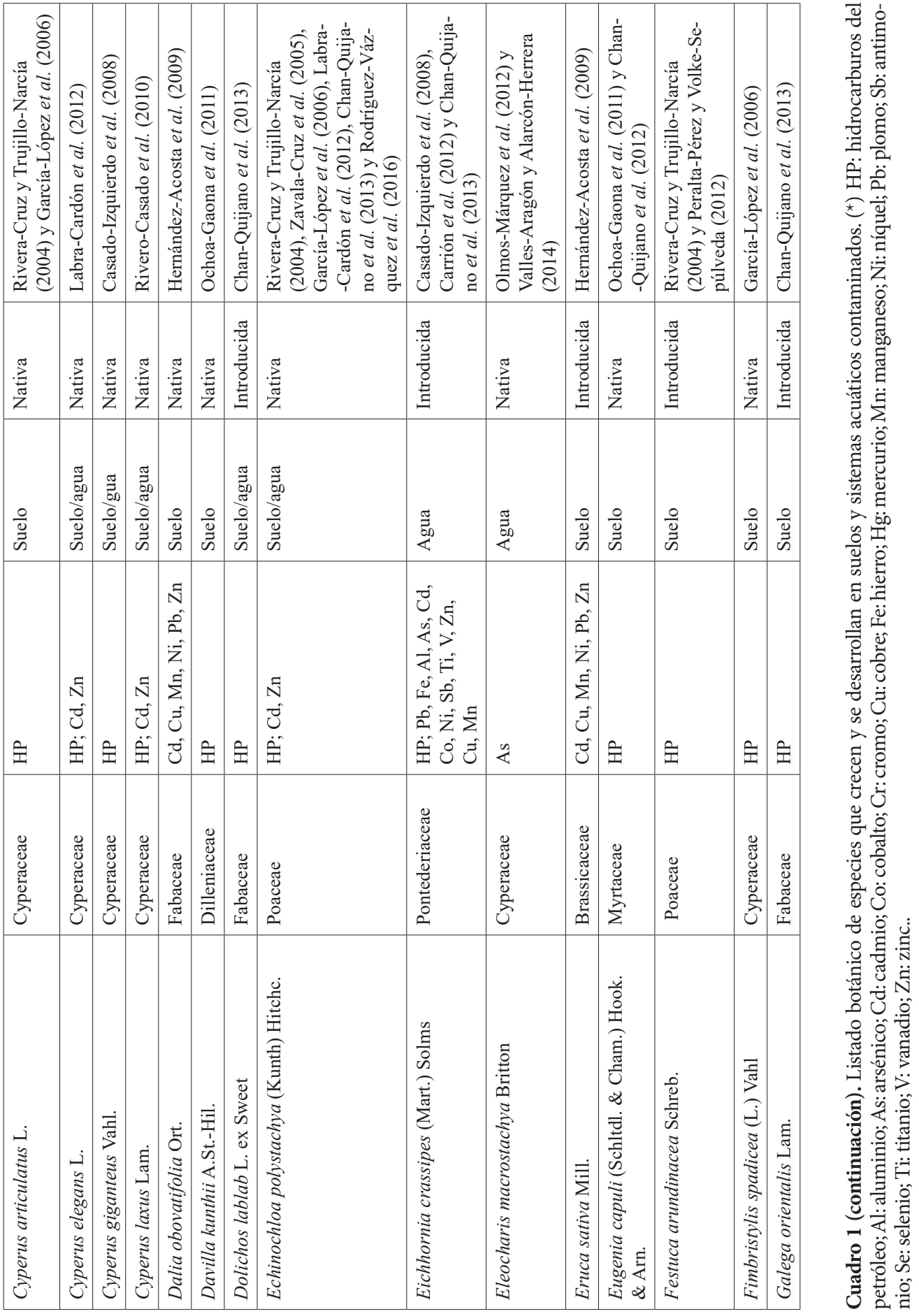




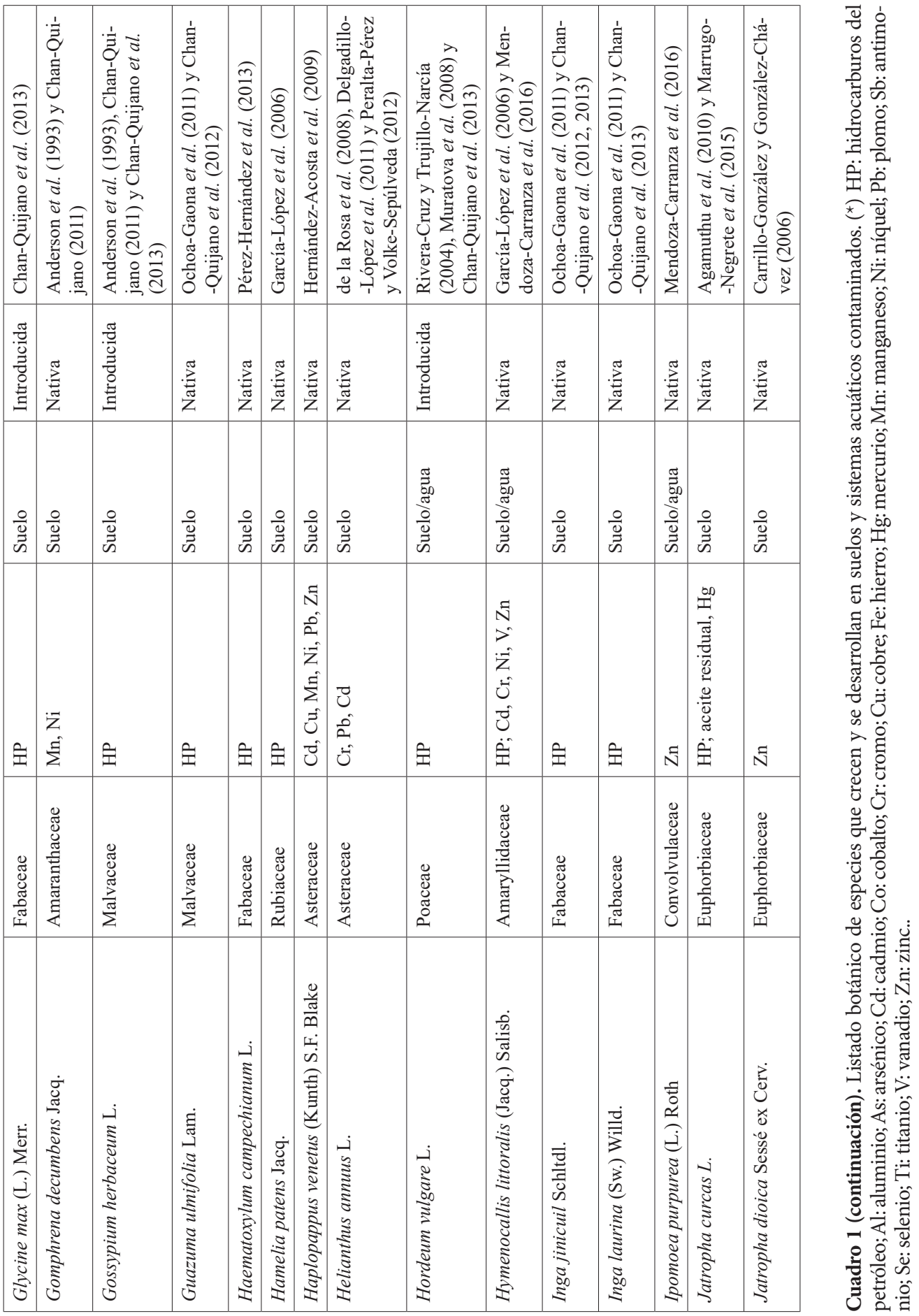




\begin{tabular}{|c|c|c|c|c|c|c|c|c|c|c|c|c|c|c|c|c|c|}
\hline 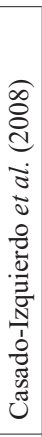 & 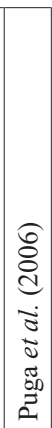 & 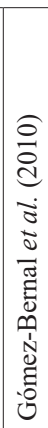 & 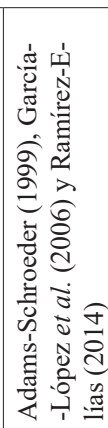 & 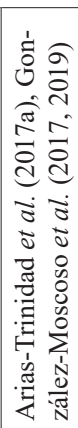 & 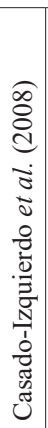 & 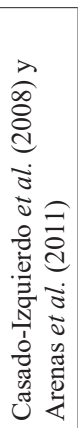 & 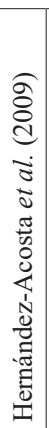 & 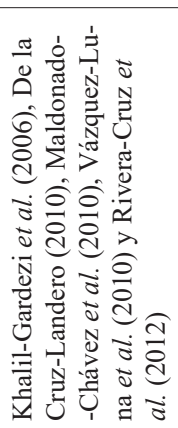 & 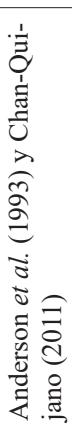 & 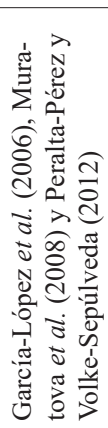 & 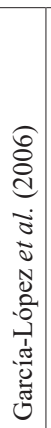 & 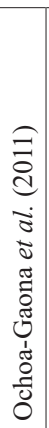 & 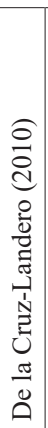 & 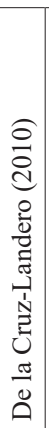 & 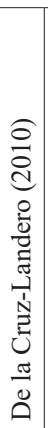 & 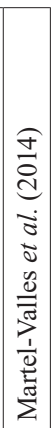 & 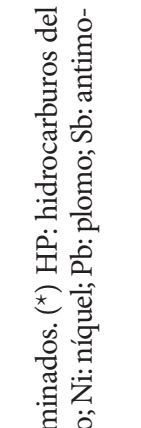 \\
\hline 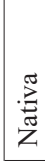 & $\begin{array}{l}\stackrel{\pi}{Z} \\
\stackrel{\Xi}{\pi} \\
Z\end{array}$ & 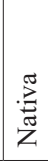 & $\begin{array}{l}\stackrel{\widetilde{Z}}{\mathbb{\Xi}} \\
\text { Z }\end{array}$ & 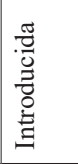 & 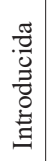 & $\begin{array}{l}\stackrel{\pi}{\overparen{\pi}} \\
\text { Z }\end{array}$ & 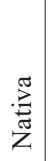 & $\begin{array}{l}\stackrel{\widetilde{E}}{Z} \\
\text { Z }\end{array}$ & 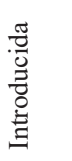 & $\begin{array}{l}\frac{\pi}{0} \\
0 \\
0 \\
0 \\
0\end{array}$ & 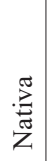 & 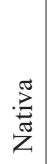 & 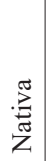 & 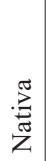 & 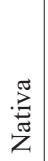 & 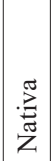 & 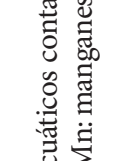 \\
\hline & $\begin{array}{l}\stackrel{0}{0} \\
\ddot{n}\end{array}$ & $\frac{O}{\stackrel{\Xi}{\Xi}}$ & 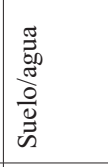 & $\begin{array}{l}\frac{0}{0} \\
\stackrel{\pi}{n}\end{array}$ & $\begin{array}{l}\frac{\pi}{0} \\
\stackrel{50}{<}\end{array}$ & 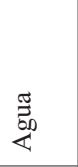 & $\begin{array}{l}\stackrel{0}{\Xi} \\
\stackrel{\Xi}{n}\end{array}$ & $\begin{array}{l}\stackrel{0}{\Xi} \\
\stackrel{\Rightarrow}{n}\end{array}$ & $\begin{array}{l}\frac{0}{0} \\
\stackrel{2}{n}\end{array}$ & $\begin{array}{l}\frac{0}{0} \\
\stackrel{2}{n}\end{array}$ & $\begin{array}{l}\stackrel{0}{\Xi} \\
\stackrel{\Xi}{n}\end{array}$ & 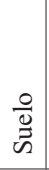 & 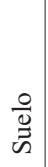 & $\begin{array}{l}\stackrel{0}{\stackrel{D}{n}} \\
\stackrel{n}{2}\end{array}$ & $\begin{array}{l}\stackrel{0}{\stackrel{\Xi}{n}} \\
\stackrel{n}{n}\end{array}$ & 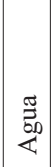 & 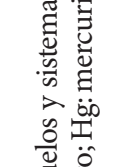 \\
\hline 主 & 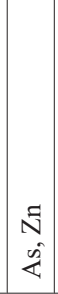 & $\begin{array}{l}\text { जี } \\
\text { \& }\end{array}$ & 主 & 主 & $\hat{\Xi}$ & $\begin{array}{l}\stackrel{00}{I} \\
\dot{\hat{\Xi}}\end{array}$ & 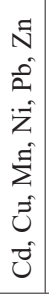 & 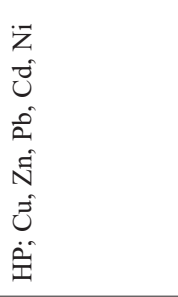 & 豆 & 主 & 王 & 引 & $\begin{array}{l}z \\
\overrightarrow{0} \\
0 \\
\hat{2} \\
\hat{N} \\
\tilde{E}\end{array}$ & $\begin{array}{l}z \\
\overrightarrow{0} \\
0 \\
\hat{2} \\
\hat{N} \\
\tilde{E}\end{array}$ & $\begin{array}{l}z \\
\overrightarrow{0} \\
0 \\
\hat{D}^{2} \\
\hat{N} \\
\tilde{E}\end{array}$ & 主 & 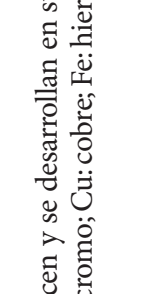 \\
\hline 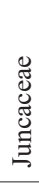 & 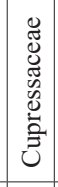 & 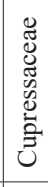 & 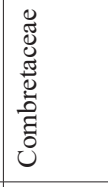 & 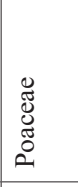 & 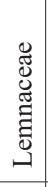 & 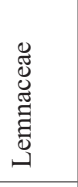 & $\begin{array}{l}\mathbb{\Xi} \\
\tilde{\Xi} \\
\tilde{\Xi} \\
0\end{array}$ & 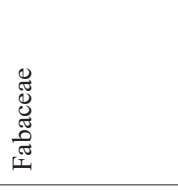 & 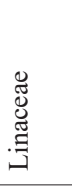 & 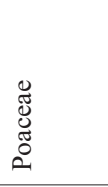 & \begin{tabular}{|c|}
$\mathscr{J}$ \\
$\tilde{J}$ \\
$\tilde{J}$ \\
$\widetilde{\pi}$ \\
त्ञ
\end{tabular} & 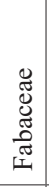 & 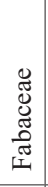 & 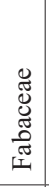 & 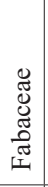 & 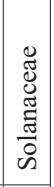 & 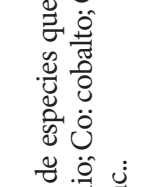 \\
\hline 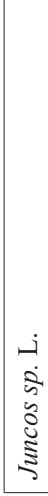 & 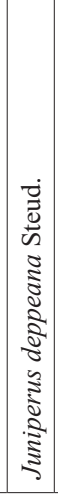 & 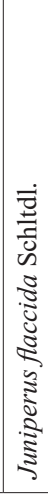 & 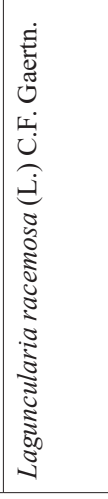 & 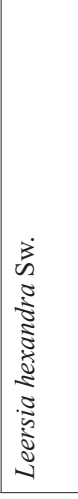 & 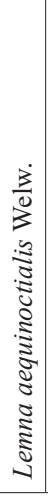 & 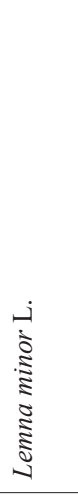 & 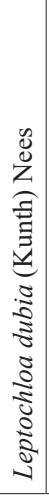 & 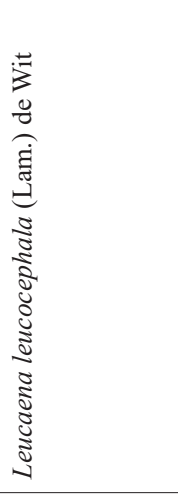 & 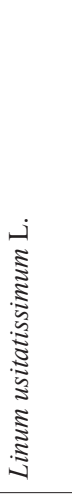 & 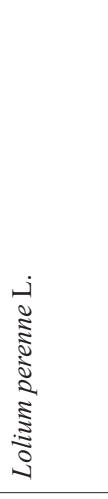 & 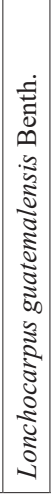 & 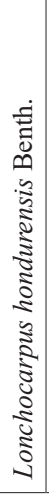 & 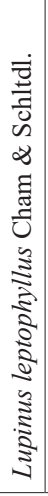 & 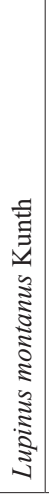 & 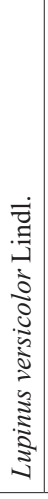 & 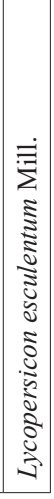 & 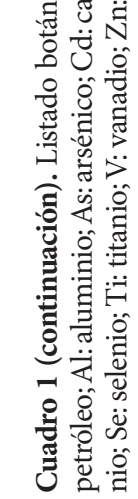 \\
\hline
\end{tabular}




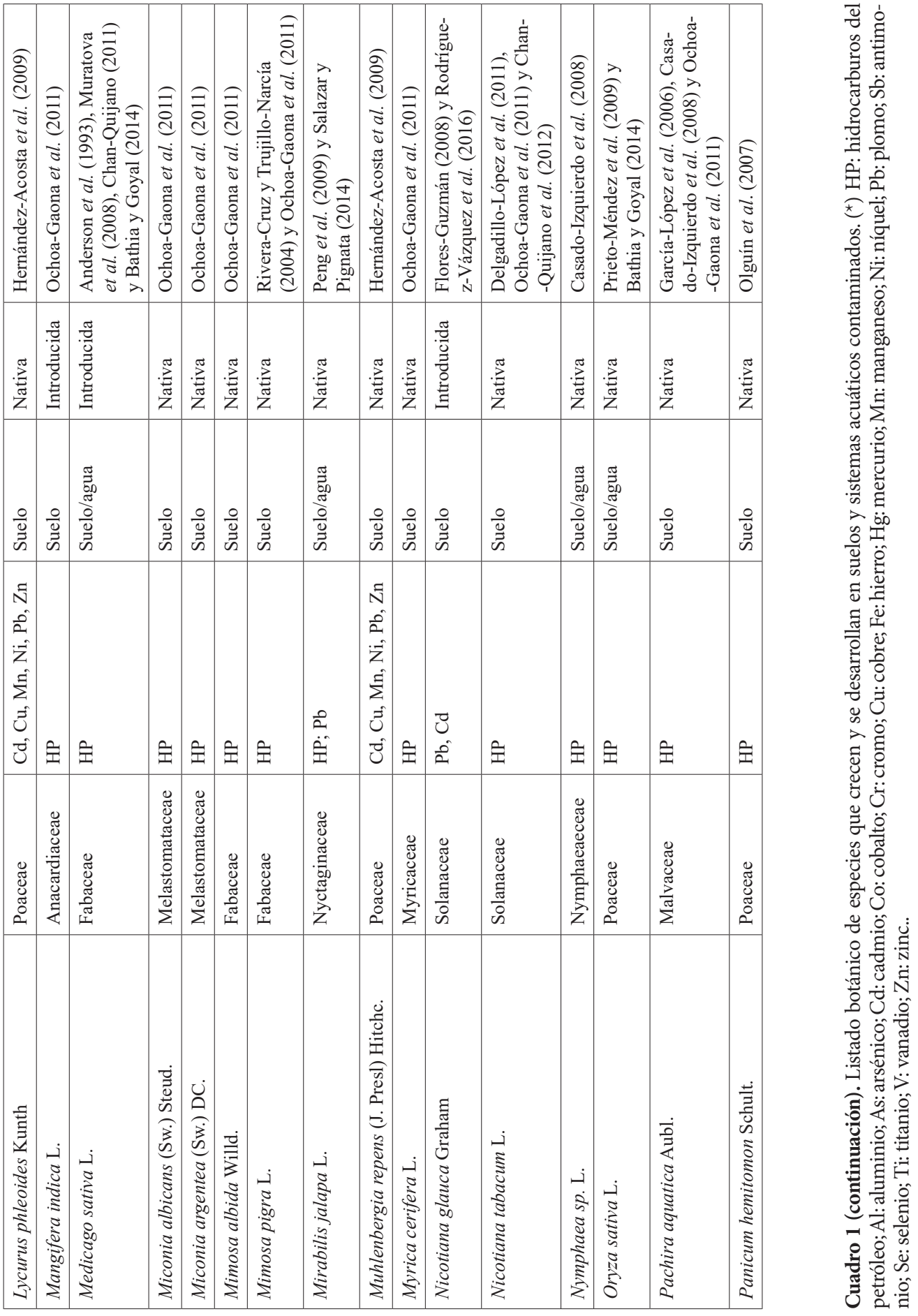




\begin{tabular}{|c|c|c|c|c|c|c|c|c|c|c|c|c|c|c|}
\hline 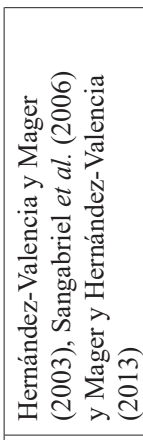 & 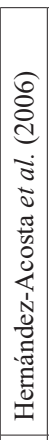 & 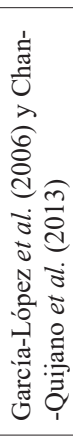 & 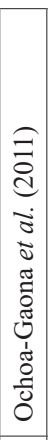 & 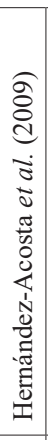 & 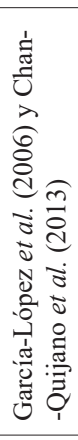 & 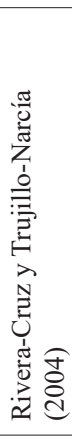 & 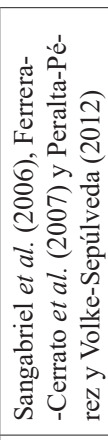 & 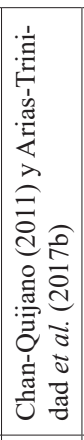 & 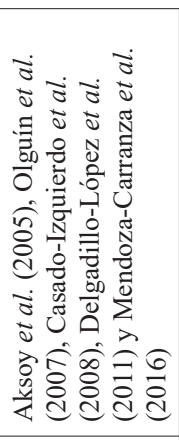 & 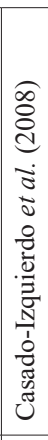 & 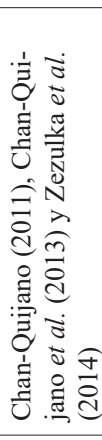 & 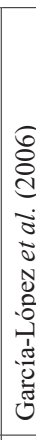 & 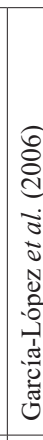 & 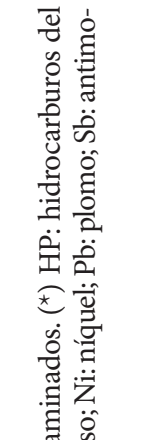 \\
\hline 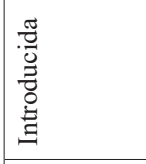 & 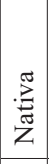 & 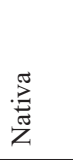 & 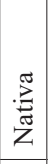 & 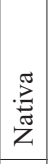 & 胥 & 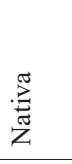 & $\begin{array}{l}\stackrel{\widetilde{J}}{\mathrm{~J}} \\
\text { Z }\end{array}$ & 总 & 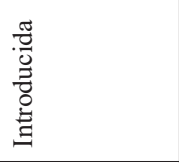 & 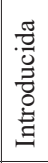 & 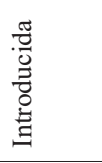 & 胥 & 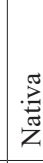 & 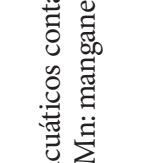 \\
\hline 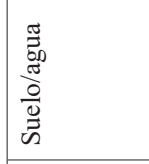 & 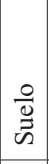 & 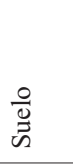 & $\begin{array}{l}\stackrel{0}{\Xi} \\
\stackrel{\Xi}{\omega}\end{array}$ & $\begin{array}{l}0 \\
\stackrel{\Xi}{\Xi} \\
\tilde{\omega}\end{array}$ & 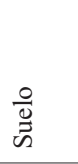 & 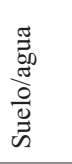 & 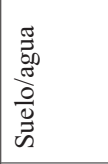 & 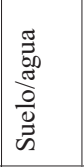 & 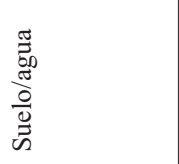 & 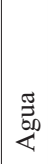 & $\begin{array}{l}\frac{0}{\varrho} \\
\ddot{n}\end{array}$ & 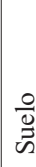 & $\begin{array}{l}0 \\
\frac{3}{3} \\
\tilde{E}\end{array}$ & 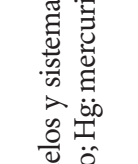 \\
\hline 结 & $引$ & 主 & 主 & 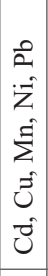 & 主 & 主 & 主 & 主 & $\begin{array}{l}\bar{z} \\
\dot{U} \\
\overrightarrow{0} \\
\dot{\theta}\end{array}$ & 主 & 主 & 主 & 主 & 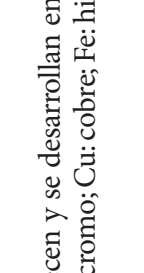 \\
\hline $\begin{array}{l}\mathbb{\Xi} \\
\mathbb{\Xi} \\
\mathbb{8} \\
\stackrel{0}{0}\end{array}$ & 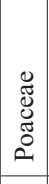 & 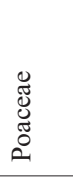 & 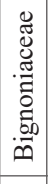 & 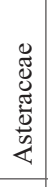 & 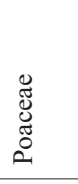 & 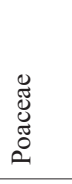 & 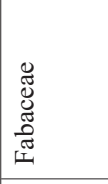 & 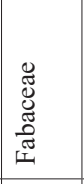 & 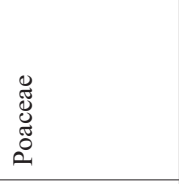 & 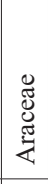 & 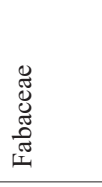 & 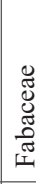 & 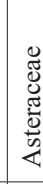 & 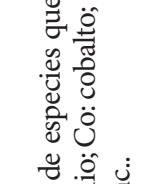 \\
\hline 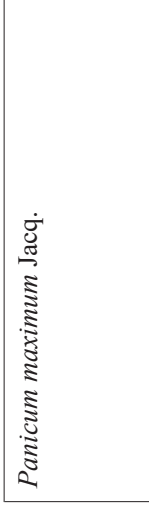 & 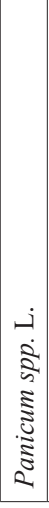 & 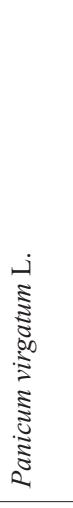 & 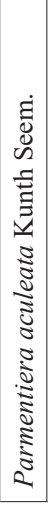 & 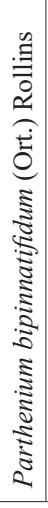 & 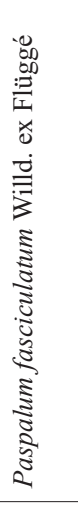 & 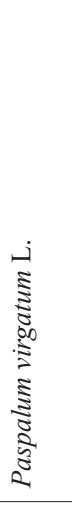 & 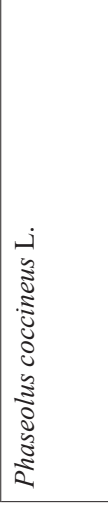 & 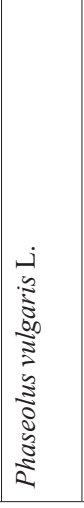 & 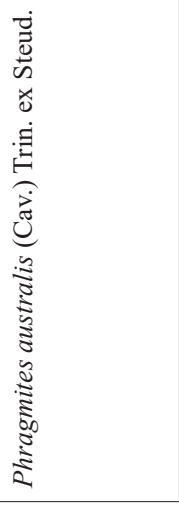 & 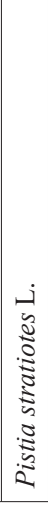 & 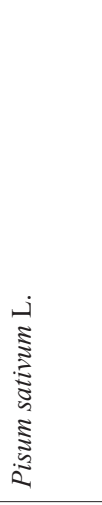 & 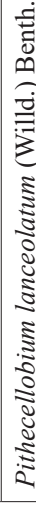 & 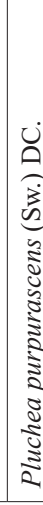 & 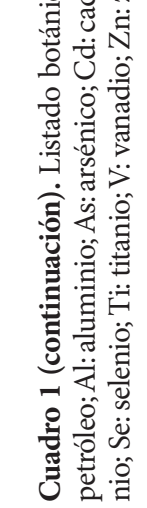 \\
\hline
\end{tabular}




\begin{tabular}{|c|c|c|c|c|c|c|c|c|c|c|c|c|c|c|c|c|c|}
\hline 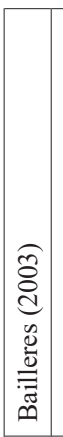 & 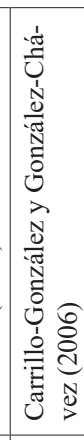 & 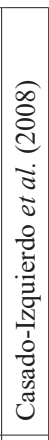 & 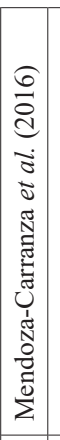 & 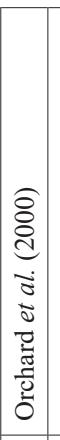 & 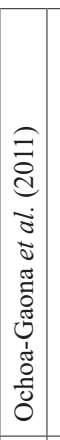 & 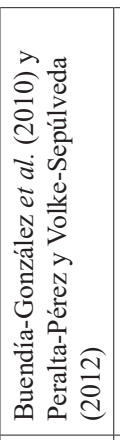 & 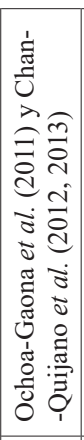 & 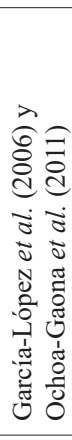 & 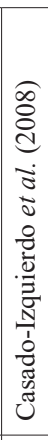 & 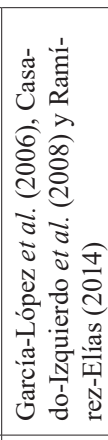 & 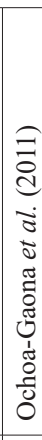 & 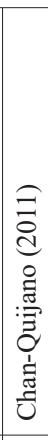 & 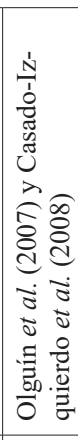 & 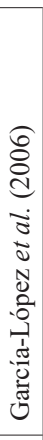 & 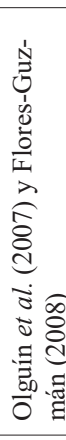 & 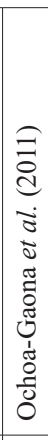 & \multirow{6}{*}{ 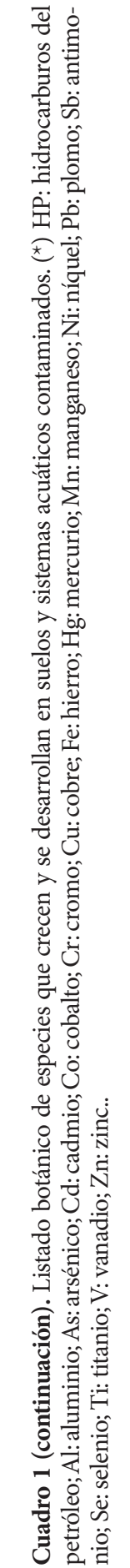 } \\
\hline 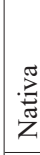 & 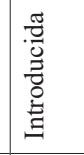 & $\begin{array}{l}\stackrel{\widetilde{D}}{己} \\
\stackrel{\mathbb{Z}}{Z}\end{array}$ & 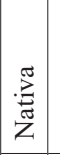 & 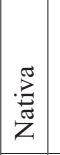 & 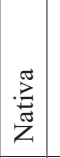 & 䓫 & 胥 & 胥 & 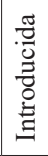 & $\begin{array}{l}\stackrel{\widetilde{Z}}{\mathrm{E}} \\
\text { Z }\end{array}$ & 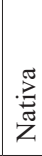 & 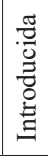 & 胥 & 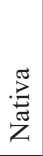 & 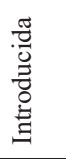 & 胥 & \\
\hline$\frac{9}{3}$ & $\frac{0}{\stackrel{\Xi}{\Xi}}$ & 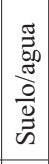 & 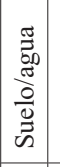 & $\begin{array}{c}0 \\
\stackrel{0}{0} \\
\tilde{n}\end{array}$ & $\begin{array}{l}\stackrel{0}{\Xi} \\
\stackrel{\tilde{n}}{2}\end{array}$ & $\begin{array}{l}\stackrel{0}{\Xi} \\
\stackrel{\tilde{\omega}}{2}\end{array}$ & 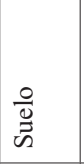 & 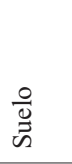 & $\begin{array}{l}\stackrel{0}{\Xi} \\
\stackrel{\Xi}{\mathscr{n}}\end{array}$ & 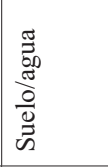 & $\begin{array}{l}\frac{\bigcirc}{\varrho} \\
\bar{\Xi}\end{array}$ & $\frac{O}{\stackrel{\Xi}{\Xi}}$ & 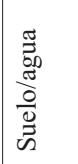 & 氶 & 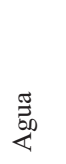 & $\begin{array}{l}\stackrel{0}{0} \\
\overrightarrow{0}\end{array}$ & \\
\hline$\frac{2}{4}$ & งี & 主 & $\begin{array}{l}\tilde{N} \\
\dot{0} \\
-\vec{z} \\
\dot{H}\end{array}$ & 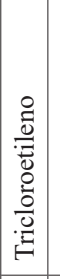 & 主 & $\begin{array}{l}\vec{z} \\
\hat{n} \\
\dot{z}\end{array}$ & 㰻 & 引 & 引 & 主 & 言 & 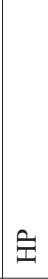 & 主 & 主 & $\begin{array}{l}\vec{z} \\
\hat{\theta} \\
\dot{N} \\
\dot{U} \\
\dot{\hat{\theta}}\end{array}$ & 主 & \\
\hline 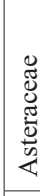 & 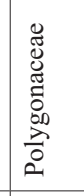 & 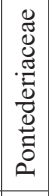 & 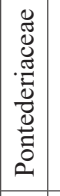 & 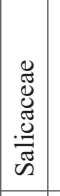 & 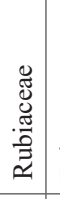 & 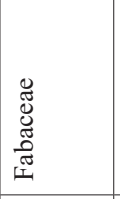 & 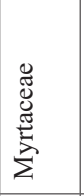 & 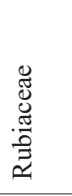 & 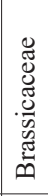 & 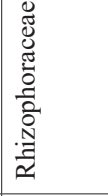 & 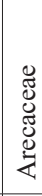 & $\begin{array}{l}\mathbb{J} \\
\mathbb{J} \\
\tilde{\Xi} \\
\stackrel{0}{0}\end{array}$ & 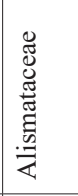 & 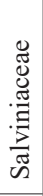 & 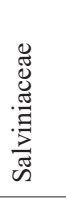 & 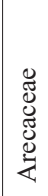 & \\
\hline 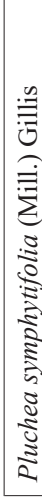 & 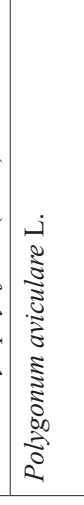 & 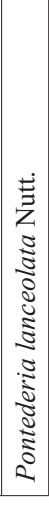 & 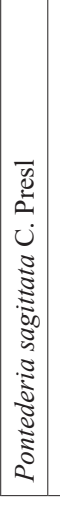 & 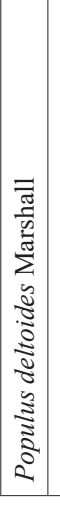 & 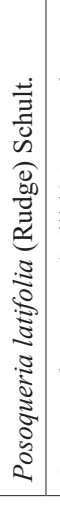 & 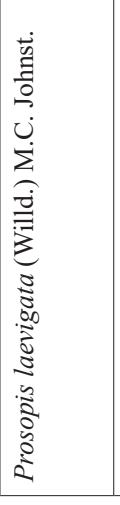 & 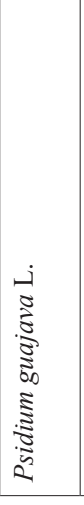 & 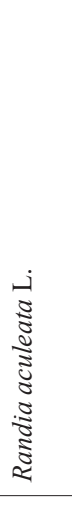 & 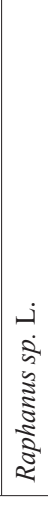 & 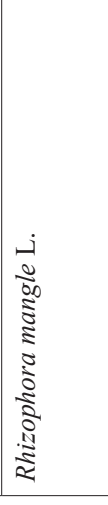 & 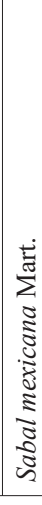 & 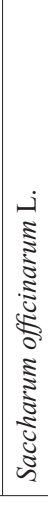 & 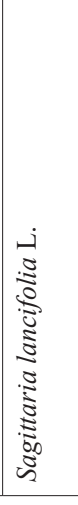 & 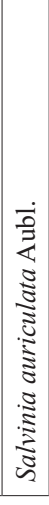 & 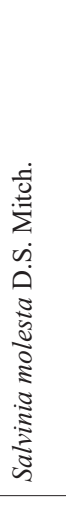 & 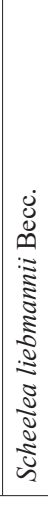 & \\
\hline
\end{tabular}




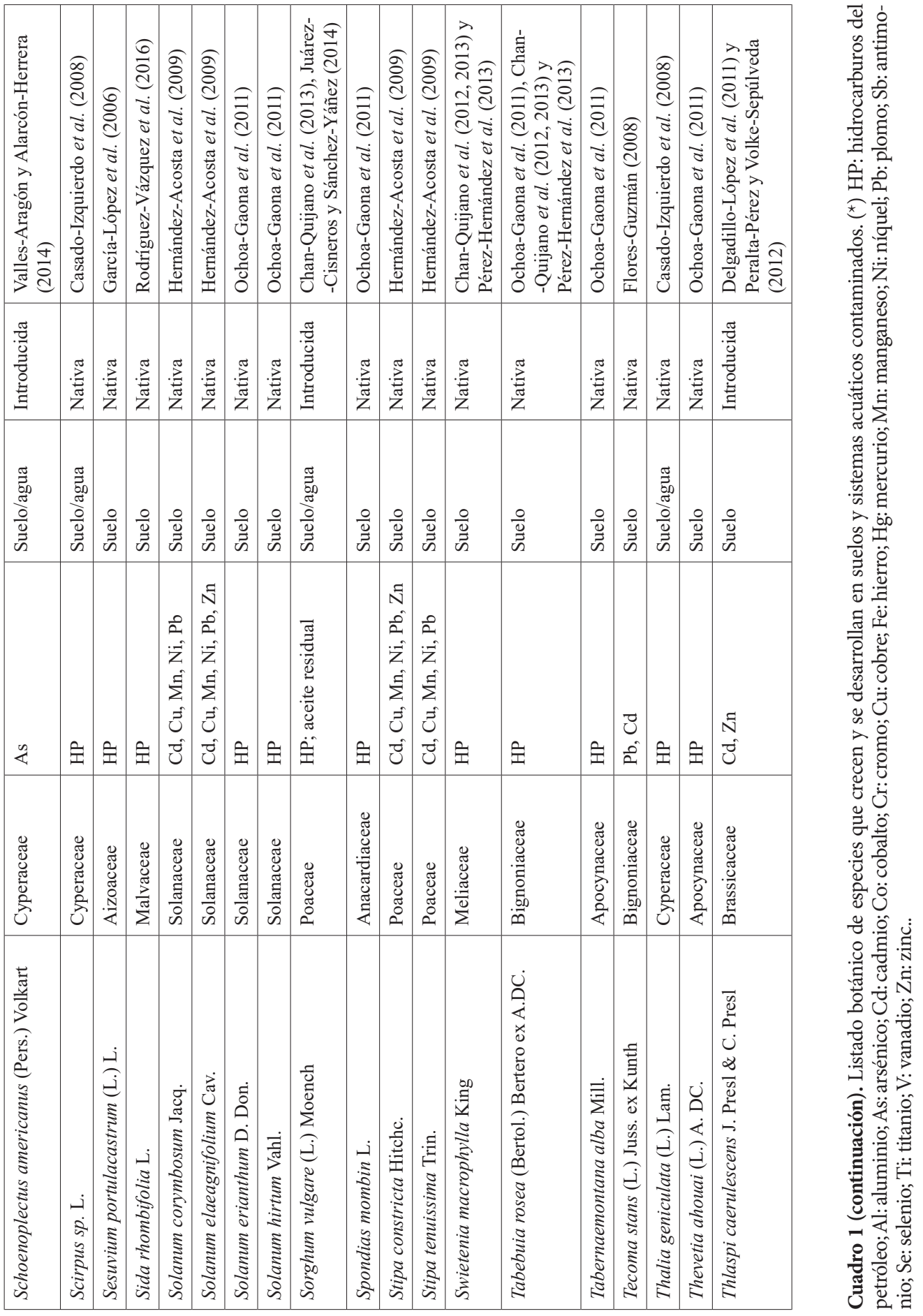




\begin{tabular}{|c|c|c|c|c|c|c|c|c|c|c|}
\hline 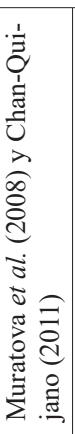 & 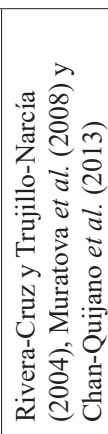 & 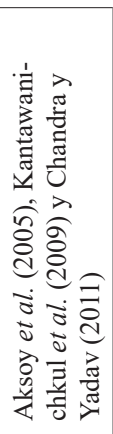 & 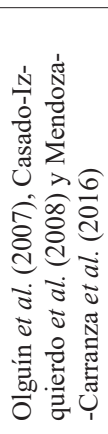 & 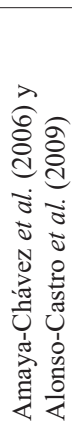 & 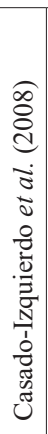 & 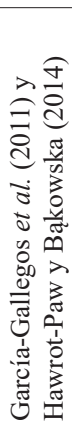 & 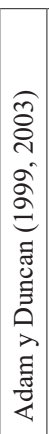 & 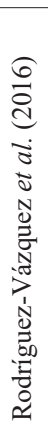 & 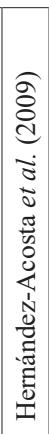 & 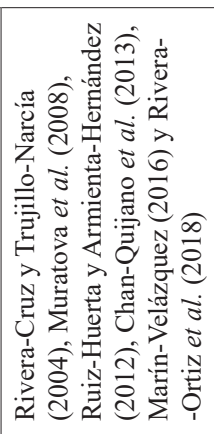 \\
\hline 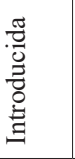 & 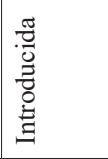 & 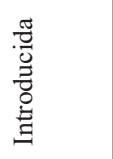 & 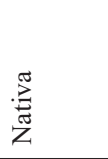 & 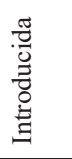 & 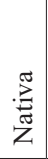 & 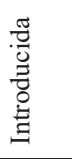 & 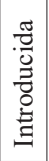 & 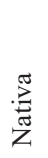 & 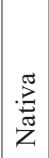 & 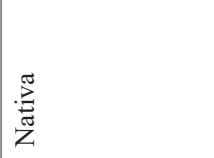 \\
\hline 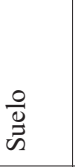 & 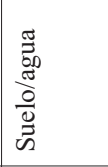 & 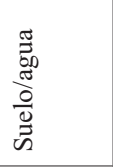 & 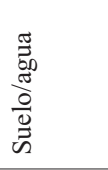 & 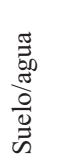 & 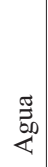 & $\begin{array}{l}\stackrel{0}{\varrho} \\
\stackrel{\Xi}{n}\end{array}$ & $\begin{array}{l}\stackrel{0}{\Xi} \\
\stackrel{\Xi}{a}\end{array}$ & $\frac{O}{\stackrel{0}{*}}$ & $\frac{O}{0}$ & 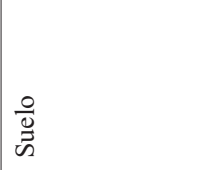 \\
\hline 急 & 主 & 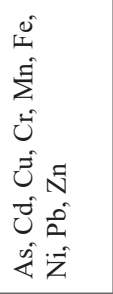 & 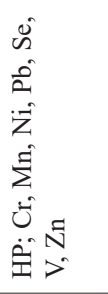 & $\begin{array}{l}\overrightarrow{2} \\
\overrightarrow{0}\end{array}$ & 引 & $\begin{array}{l}\overrightarrow{0} \\
\dot{\tilde{D}} \\
\stackrel{0}{0} \\
\stackrel{0}{0}\end{array}$ & 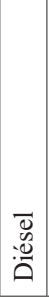 & 主 & 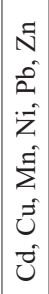 & 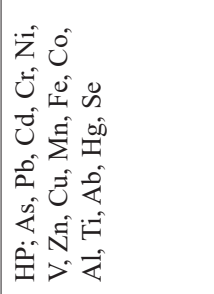 \\
\hline 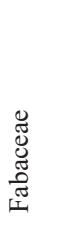 & 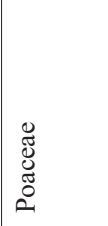 & 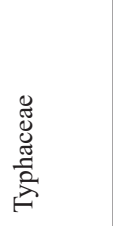 & 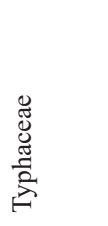 & 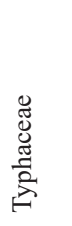 & 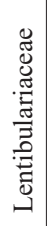 & 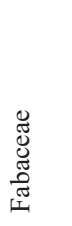 & 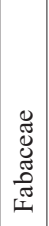 & 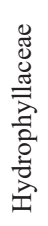 & 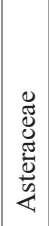 & $\begin{array}{l}\mathscr{J} \\
\mathbb{J} \\
\tilde{D} \\
\stackrel{0}{0}\end{array}$ \\
\hline 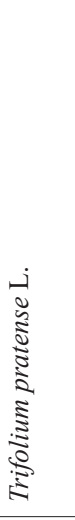 & 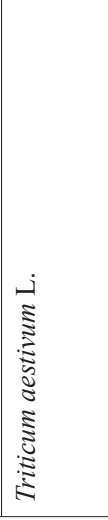 & 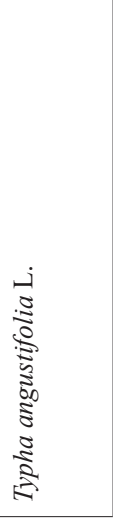 & 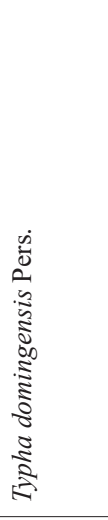 & 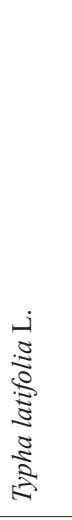 & 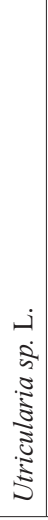 & $\frac{\dot{1}}{\tilde{D}}$ & 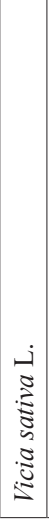 & 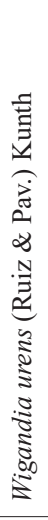 & 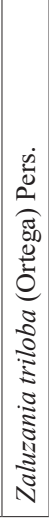 & 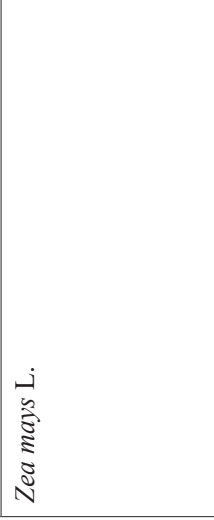 \\
\hline
\end{tabular}




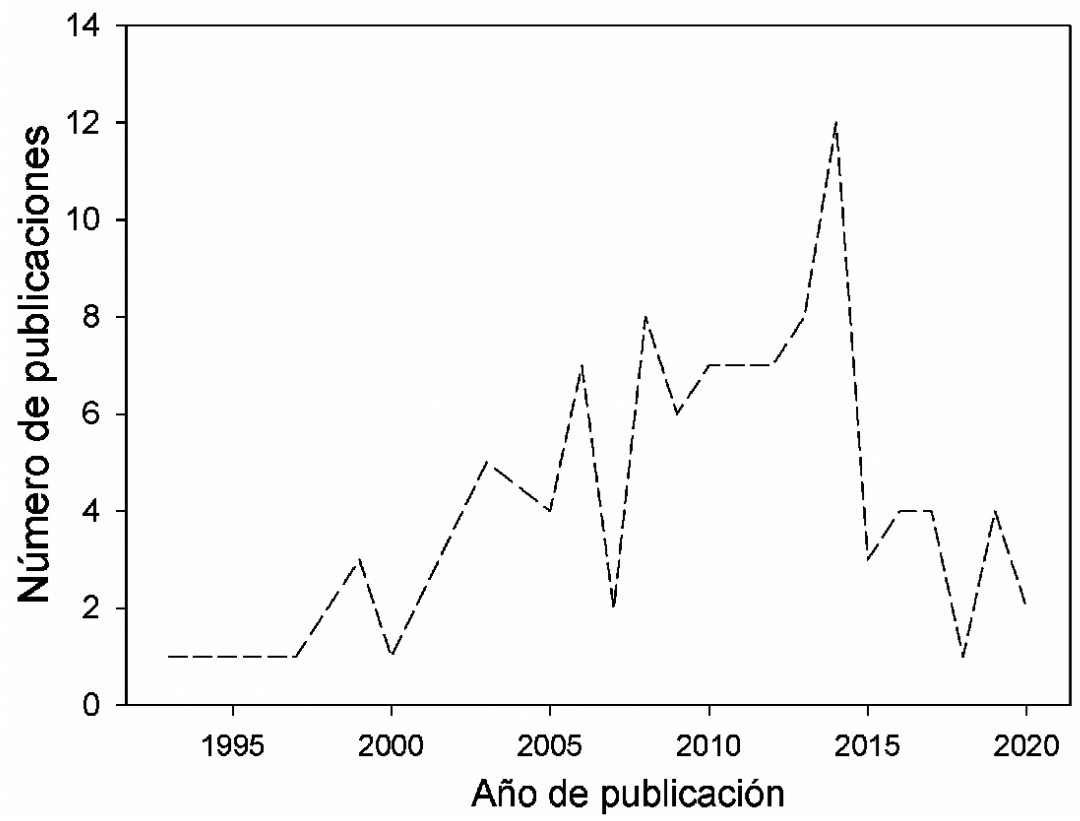

Figura 2. Número de publicaciones por año sobre especies vegetales utilizadas en procesos de remediación de ambientes contaminados.

\section{Discusión}

Chan-Quijano et al. (2013) reportaron 129 especies, pertenecientes a 47 familias botánicas. En este trabajo se agregan 45 plantas más al listado, así como seis familias botánicas. La actualización de esta lista botánica representa un esfuerzo por reconocer la diversidad de especies con capacidad de crecer y desarrollarse en zonas contaminadas con HP y metales pesados, aumentando considerablemente el número de especies y clasificándolas entre nativas e introducidas, lo que puede constituir un criterio de selección en el uso de especies en programas de biorremediación.

Las especies reportadas en el listado botánico tiene la capacidad de eliminar, biotransformar o bioacumular los contaminantes a través de la fitorremediación y/o biorremediación apoyados por los microorganismos que viven en la zona radical (Schnoor et al. 1995, Rivera-Cruz et al. 2005, Peralta-Pérez y Volke-Sepúlveda 2012, Bathia y Goyal 2014).

\section{Desarrollo de plantas en áreas contaminadas}

En la remediación de los suelos contaminados se ha estado utilizando plantas herbáceas, mayormente gramíneas; en el caso de los sistemas acuáticos se aplican hidrófitas (Alarcón y Ferrera-Cerrato 2013). Sin embargo, para las zonas terrestres se ha comenzado a utilizar la remediación asistida con especies arbóreas nativas con capacidad de tolerar a los contaminantes; esto puede ser parte de las estrategias para la recuperación de las áreas afectadas por los derrames de petróleo y, a su vez, aplicar directrices de manejo para la protección de los suelos y cuerpos de agua (Vázquez-Yanes et al. 1999, Hou y Ok 2019).

A diferencia de los listados botánicos realizados nivel local (Ederra 1997, Manios et al. 2003, García-López et al. 2006, Ochoa-Gaona et al. 2011), el presente trabajo ofrece un listado a mayor escala.

Específicamente, existen varias especies de plantas que poseen un sistema de raíces fibro- 
sas que proveen una gran superficie para los microorganismos de la rizosfera, en donde se ha probado el establecimiento de especies con capacidad para remediar suelos contaminados; entre estas especies destacan algunas de la familia Poaceae como Sorghum vulgare, Andropogon gerardii, Panicum virgatum, Lolium perenne, Echinochloa polystachya y Paspalum fasciculatum.

En cuanto a las plantas acuáticas, ellas tienen la capacidad de realizar una rizofiltración por sus raíces logrando metabolizar, biodegradar o bioacumular los contaminantes del agua. El proceso que desarrollan para eliminar el contaminante del agua es por medio de la nitrificación y desnitrificación debido a las reacciones microbianas que se llevan a cabo alrededor de las raíces (Ferrera-Cerrato et al. 2006, PérezLópez 2009). Algunas de las especies con esta capacidad se encuentran en la familia Pontederiaceae, Eichhornia crassipes, por ejemplo.

Estas plantas pueden aprovecharse para establecer objetivos para reducir los riesgos ambientales de las áreas contaminadas (BP 2013). Sin embargo, para un enfoque más holístico en la remediación de los suelos y aguas contaminadas, se deben evaluar los procesos de adsorción, transporte y translocación, así como la bioacumulación, la transformación y la mineralización de los contaminantes por parte de las plantas (Pandey et al. 2014), para entonces obtener una remediación sustentable que sea económica, ambientalmente viable y socialmente aceptada. De igual forma, se debe proponer un diseño experimental para el uso de especies vegetales nativas de la zona para los procesos asistidos de la remediación y con ello, lograr a mediano y largo plazo la restauración del área.

$\mathrm{Al}$ igual, en los estudios de remediación de las zonas afectadas por contaminantes se debe incluir las interacciones complejas en donde participan la planta, los microorganismos, el suelo, el agua y los contaminantes, para identificar cuáles son los efectos de la recuperación o restauración del sitio. Sin embargo, se conoce poco sobre los mecanismos de la remediación, del desempeño, de los procesos moleculares y de la ponderación de las etapas en donde los contaminantes son absorbidos y transformados hasta su destino final (López-Martínez et al. 2005). Con esta revisión queda claro que hace falta mucho trabajo por realizar, el cual permitirá proponer soluciones a los problemas de la contaminación y la eventual recuperación del suelo y de los sistemas acuáticos contaminados.

\section{Conclusiones}

La valoración de la calidad de desarrollo de las especies vegetales en ambientes contaminados ha sido de utilidad como alternativa de manejo técnico-biológico para la remediación de estos sitios. El presente listado botánico podrá ser utilizado como referencia de la gran cantidad de plantas que funcionan en la remediación de suelos y sistemas acuáticos contaminados por HP y metales pesados.

A pesar de que se registraron 174 plantas, aún son necesarios los estudios sobre la capacidad de tolerancia y concentración de los contaminantes en las especies vegetales, ya que no todas las plantas tienen esta capacidad. De igual forma, se debe estudiar los procesos fisiológicos pues las características físicas y químicas del suelo o del agua influyen en los procesos de crecimiento y desarrollo de las plantas y al mismo tiempo, en el proceso de remediación.

De igual forma, son necesarios trabajos que contribuyan a entender las estrategias de aclimatación que siguen las diferentes especies, lo que contribuirá a entender su capacidad de tolerancia a diferentes contaminantes, así como su rol en posibles estrategias de biorremediación y/o fitorremediación.

\section{Referencias}

Adam, G; Duncan, H. 1999. Effect of diesel fuel on growth of selected plant species. Environmental Geochemistry and Health (21):353-357. DOI: https://doi. org/10.1023/A:1006744603461.

Adam, G; Duncan, H. 2003. The effect of diesel fuel on common vetch (Vicia faba 
L.) plants. Environmental Geochemistry Health (25):123-130. DOI: https://doi. org/10.1023/A:1021228327540.

Adams, RH; Álvarez-Ovando, AL; Castañón, G. 2015. Effect of hydrocarbon concentration of pasture production (Brachiaria humidicola) in Texistepec, Veracruz. FYTON: International Journal of Experimental Botany 84(1):222-232.

Adams-Schroeder, RH. 1999. Recuperación con mangle blanco (Laguncularia racemosa) de áreas impactadas por hidrocarburos y su manejo como agrosilvo-ecosistema en la zona costera de Huimanguillo y Cárdenas, Tabasco (en línea). Tabasco, México, UJAT. 77 p. Informe final. Proyecto M076-SNIB-CONABIO. Consultado 13 nov. 2019. Disponible en http:// www.conabio.gob.mx/institucion/proyectos/ resultados/InfM076.pdf.

Adki, VS; Jadhav, JP; Bapat, VA. 2013. At the cross roads of environmental pollutants and phytoremediation: a promising bio remedial approach. Journal of Plant Biochemistry and Biotechnology 23(2):125-140. DOI: http://doi. org/10.1007/s13562-013-0250-6.

Agamuthu, P; Abioye, OP; Aziz, AA. 2010. Phytoremediation of soil contaminated with used lubricating oil using Jatropha curcas. Journal of Hazardous Materials 179(1-3):891-894. DOI: https://doi.org/10.1016/j.jhazmat.2010.03.088.

Aksoy, A; Duman, F; Sezen, G. 2005. Heavy metal accumulation and distribution in narrow-leaved cattail (Typha angustifolia) and common reed (Phragmites australis). Journal of Freshwater Ecology 20(4):783-785. DOI: http:// doi.org/10.1080/02705060.2005.9664806.

Alarcón, A; Ferrera-Cerrato, F. 2013. Biorremediación de suelos y aguas contaminadas con compuestos orgánicos e inorgánicos. México DF, México, Trillas. 333 p.

Alonso-Castro, AJ; Carranza-Álvarez, C; Alfaro-de la Torre, MC; Chávez-Guerrero, L; García-De la Cruz, RF. 2009. Removal and accumulation of cadmium and lead by Typha latifolia exposed to single and mixed metal solutions. Archives of Environmental Contam- ination Toxicology (57):688-696. DOI: http:// doi.org/10.1007/s00244-009-9351-6.

Amaya-Chávez, A; Martínez-Tabche, L; LópezLópez, E; Galar-Martínez, M. 2006. Methyl parathion toxicity to and removal efficiency by Typha latifolia in water and artificial sediments. Chemosphere 63(7):1124-1129. DOI: http:// doi.org/10.1016/j.chemosphere.2005.09.049.

Anderson, TA; Guthrie, EA; Walton, BT. 1993. Bioremediation in the rhizosphere: plant roots and associated microbes clean contaminated soil. Environmental Science Technology 27(13):2630-2636. DOI: http://doi. org/10.1021/es00049a001.

Arenas, AD; Lué-Merú, M; Torres, G. 2011. Evaluación de la planta Lemna minor como biorremediadora de aguas contaminadas con mercurio. Avances en Ciencias e Ingeniería 2(3):1-11.

Arias-Trinidad, A; Rivera-Cruz, MC; Roldán-Garrigós, A; Aceves-Navarro, LA; Quintero-Lizaola, R; Hernández-Guzmán, J. 2017a. Uso de Leersia hexandra (Poaceae) en la fitorremediación de suelos contaminados con petróleo fresco e intemperizado. Revista Biología Tropical 65(1):21-30.

Arias-Trinidad, A; Rivera-Cruz, MC; Trujillo-Narcía, A. 2017b. Fitotoxicidad de un suelo contaminado con petróleo fresco sobre Phaseolus vulgaris L. (Leguminosae). Revista Internacional de Contaminación Ambiental 33(3):411-419. DOI: http://doi.org/10.20937/ RICA.2017.33.03.05.

Armienta, MA; Ongley, LK; Rodríguez, R; Cruz, O; Mango, H; Villaseñor, G. 2008. Arsenic distribution in mesquite (Prosopis laevigata) and huizache (Acacia farnesiana) in the Zimapán mining area, México. Geochemistry: exploration, environment, analysis 8(2):191-197. DOI: http://doi.org/10.1144/1467-7873/07-144.

Bailleres, A. 2003. Hacia un desarrollo sustentable. Informe anual ambiental 2002 (en línea). Ciudad de México, México, Industrias Peñoles SAB de CV. 15 p. Consultado 6 ene. 2021. Disponible en http://www.penoles.com.mx. 
Bathia, M; Goyal, D. 2014. Analyzing remediation potential of wastewater through wetland plants: a review. Environmental Progress and Sustainable Energy 33(1):9-27. DOI: http://doi. org/10.1002/ep.11822.

Bautista-García, RA; Sánchez-Reyes, A. 2009. Fitorremediación de metales pesados y microorganismos. Revista electrónica de la Agencia de Medio Ambiente 9(16):1-6.

BP (British Petroleum Company). 2013. Sustainability review 2012: building a stronger, safer BP (en línea). Londres, Reino Unido. Consultado 6 ene. 2021. Disponible en http://www. bp.com.

Buendía-González, L; Orozco-Villafuerte, O; Estrada-Zúñiga, ME; Barrera-Díaz, CE; Vernon-Carter, EJ; Sosa-Cruz, F. 2010. Acumulación in vitro de plomo y níquel en plántulas de mezquite (Prosopis laevigata). Revista Mexicana de Ingeniería Química 9(1):1-9.

Burges, A; Epelde, L; Blanco, F; Becerril, JM; Garbisu, C. 2017. Ecosystem services and plant physiological status during endophyte-assisted phytoremediation of metal contaminated soil. Science of the Total Environment (584585):329-338. DOI: http://dx.doi.org/10.1016/j. scitotenv.2016.12.146.

Carrillo-González, R; González-Chávez, MCA. 2006. Metal accumulation in wild plants surrounding mining wastes. Environmental Pollution 144(1):84-92. DOI: http://doi. org/10.1016/j.envpol.2006.01.006.

Carrión, C; Ponce-de León, C; Cram, S; Sommer, I; Hernández, M; Vanegas, C. 2012. Potential use of water hyacinth (Eichhornia crassipes) in Xochimilco for metal phytoremediation. Agrociencia 46(6):609-620.

Casado-Izquierdo, JM; Hernández-Juárez, M; Ortiz-Pérez, MA; Oropeza-Orozco, O; Quintero-Pérez, JA; Sánchez-Salazar, T; Sommer-Cervantes, I. 2008. Validación en campo de variables e índices de vulnerabilidad en sitios ambientalmente sensibles a derrames de hidrocarburos en la región de Coatzacoalcos. Ciudad de México, México, INE. 65 p.
Chandra, R; Yadav, S. 2011. Phytoremediation of $\mathrm{Cd}, \mathrm{Cr}, \mathrm{Cu}, \mathrm{Mn}, \mathrm{Fe}, \mathrm{Ni}, \mathrm{Pb}$ and $\mathrm{Zn}$ from aqueous solution using Phragmites cummunis, Typha angustifolia and Cyperus esculentus. International Journal of phytoremediation 13(6):580-591. DOI: http://doi.org/10.1080/15 226514.2010.495258.

Chan-Quijano, JG. 2011. Aplicación de técnicas de germinación a semillas de especies leñosas nativas promisorias para la fitorremediación de suelos contaminados por hidrocarburos en Tabasco, México. Tesis Lic. Quintana Roo, México, Universidad de Quintana Roo. 106 p.

Chan-Quijano, JG; Cach-Pérez, MJ; Rodríguez-Robles, U.2020. Phytoremediation of soils contaminated by hydrocarbon. In Shmaefsky, BR (ed.). Phytoremediation: in situ aplications. Berna, Suiza, Springer. p. 83-101. DOI: http:// doi.org/10.1007/978-3-030-00099-8 3 .

Chan-Quijano, JG; Jarquín-Sánchez, A; Ochoa-Gaona, S; Bautista-Zúñiga, F; Martínez-Zurimendi, P; López-Chávez, MY. 2013. Especies vegetales útiles para fitorremediar suelos contaminados con hidrocarburos totales del petróleo: un apoyo para la restauración ecológica. Boletín de la Red Iberoamericana y del Caribe de Restauración Ecológica 7(3):11-14.

Chan-Quijano, JG; Jarquín-Sánchez, A; Ochoa-Gaona, S; Martínez-Zurimendi, P; López-Jiménez, LN; Lázaro-Vázquez, A. 2015. Guidelines for the remediation of contaminated soil with oil. Teoría y Praxis (17):123-144. DOI: https://doi.org/10.22403/UQROOMX/ TYP17/05.

Chan-Quijano, JG; Ochoa-Gaona, S; Gutiérrez-Aguirre, MA; Pérez-Hernández, I. 2012. Aplicación de técnicas de germinación a semillas de especies leñosas nativas promisorias para la fitorremediación de suelos contaminados por hidrocarburos en Tabasco, México. Boletín Divulgativo de la Red Iberoamericana y del Caribe de Restauración Ecológica 6(1):1214.

CONABIO (Comisión Nacional para el Conocimiento y Uso de la Biodiversidad, México). 2019. Herbario virtual (en línea). Ciudad de 
México, México. Consultado 8 dic. 2019. Disponible en http://www.conabio.gob.mx/otros/ cgi-bin/herbario.cgi.

De la Cruz-Landero, N. 2010. Especies de leguminosas como fitorremediadoras en suelos contaminados. Tesis D.Sc. Ciudad de México, México, Colegio de Postgraduados. 145 p.

de la Rosa, G; Cruz-Jiménez, G; Cano-Rodríguez, I; Fuentes-Ramírez, R; Gardea-Torresdey, JL. 2008. Efecto de la edad de la planta y presencia de SS-EDDS en la tolerancia y absorción de Cr (III) por Helianthus annuus. Revista Mexicana de Ingeniería Química 7(3):243-251.

Delgadillo-López, AE; González-Ramírez, CA; Prieto-García, F; Villagómez-Ibarra, JR; Acevedo-Sandoval, O. 2011. Phytoremediation: an alternative to eliminate pollution. Tropical and Subtropical Agroecosystems 14(2):597- 612.

Díaz-Martínez, ME; Alarcón, A; Ferrera-Cerrato, R; Almaraz-Suarez, JJ; García-Barradas, O. 2013. Crecimiento de Casuarina equisetifolia (Casuarinaceae) en suelo con diésel, y aplicación de bioestimulación y bioaumentación. Revista Biología Tropical 61(3):1039-1052.

Ederra, IA. 1997. Botánica ambiental aplicada, las plantas y el equilibrio ecológico de nuestra tierra. Pamplona, España, Universidad de Navarra. $212 \mathrm{p}$.

Fernández-Linares, LC; Rojas-Avelizapa, NG; Roldán-Carrillo, TG; Ramírez-Islas, ME; Zegarra-Martínez, HG; Uribe-Hernández, R; Reyes-Ávila, RJ; Flores-Hernández, D; Arce-Ortega, JM. 2006. Manual de técnicas de análisis de suelo aplicadas a la remediación de sitios contaminados. México DF, México, INE. $179 \mathrm{p}$.

Ferrera-Cerrato, R; Alarcón, A; Mendoza, M; Sangabriel, W; Trejo-Aguilar, D; Cruz-Sánchez, JS; López-Ortiz, C; Delgadillo-Martínez, J. 2007. Fitorremediación de un suelo contaminado con combustóleo utilizando Phaseolus coccineus y fertilización orgánica e inorgánica. Agrociencia 41(8):817-826.

Ferrera-Cerrato, R; Rojas-Avelizapa, NG; Poggi-Varaldo, HM; Alarcón, A; Cañizares-Villan- ueva, RO. 2006. Procesos de biorremediación de suelo y agua contaminados por hidrocarburos del petróleo y otros compuestos orgánicos. Revista Latinoamericana de Microbiología 48(2):179-187.

Flores-Guzmán, CM. 2008. Determinación de bioacumulación de plomo y cadmio mediante absorción atómica en Alyssum murale, Tecoma stans y Nicotiana glauca colectadas en la comunidad minera de Maconí. Tesis Mg.Sc. Querétaro, México, Universidad Autónoma de Querétaro.61 p.

Flores-Tavizón, E; Alarcón-Herrera, M; González-Elizondo, S; Holguín, E. 2003. Arsenic tolerating plants from mine sites and hot springs in the semi-arid region of Chihuahua, Mexico. Acta Biotechnologica 23(2):113-119. DOI: http://doi.org/10.1002/abio.200390016.

García-Gallegos, E; Hernández-Acosta, E; García-Nieto, E; Acevedo-Sandoval, OA. 2011. Lead content and translocation in oats ( $A v$ ena sativa, L.) and broad bean (Vicia faba, L.) in contaminated soil. Revista Chapingo (Serie Ciencias Forestales y del Ambiente) 17(1): 19-29. DOI: http://doi.org/10.5154/r.rchscfa.2010.02.002.

García-López, E; Zavala-Cruz, J; Palma-López, DJ. 2006. Caracterización de las comunidades vegetales en un área afectada por derrames de hidrocarburos. Terra Latinoamericana 24(1):17-26.

Gardi, C; Angelini, M; Barceló, S; Comerma, J; Cruz-Gaistardo, C; Encina-Rojas, A; Jones, A; Krasilnikov, P; Mendonça, ML; Montanarella, L; Muñiz, O; Schad, P; Vara-Rodríguez, MI; Vargas, R. 2014. Atlas de suelos de América Latina y el Caribe (en línea). Luxemburgo, Luxemburgo, UE. Consultado 6 ene. 2021. Disponible en http://www.fao.org/agronoticias/agro-publicaciones/agro-publicacion-detalle/es/c/239323/.

Global Plants. 2020. Global Plant on Jstor (en línea). Consultado 16 jul. 2020. Disponible en https://plants.jstor.org/.

Gómez-Bernal, JM; Santana-Carrillo, J; Romero-Martín, F; Armienta-Hernández, MA; Morton-Bermea, O; Ruíz-Huerta, EA. 2010. Plantas 
de sitios contaminados con desechos mineros en Taxco, Guerrero, México. Boletín de la Sociedad Botánica de México (87):131-133.

González, I; Muena, V; Cisternas, M; Neaman, A. 2008. Acumulación de cobre en una comunidad vegetal afectada por contaminación minera en el valle de Puchuncaví, Chile central. Revista Chilena de Historia Natural 81(2):279291. DOI: http://doi.org/10.4067/S0716078X2008000200010.

González-Moscoso, M; Rivera-Cruz, MC; Delgadillo-Martínez, J; Lagunes-Espinoza, LC. 2017. Análisis de crecimiento y producción vegetal de Leersia hexandra Swartz en el trópico húmedo mexicano en función de petróleo y surfactante. Polibotánica (43):177-196. DOI: http://doi.org/10.18387/polibotanica.43.8.

González-Moscoso, M; Rivera-Cruz, MC; Trujillo-Narcía, A. 2019. Decontamination of soil containing oil by natural attenuation, phytoremediation and chemical desorption. International Journal on Phytoremediation 21(8):768776. DOI: http://doi.org/10.1080/15226514.20 19.1566879 .

Hawrot-Paw, M; Bąkowska, T. 2014. Growth and development of selected plant species in the phytoremediation of diesel oil contaminated soil. Environment Protection Engineering 40(4):5-13. DOI: http://doi.org/10.5277/ epe140401.

Hernández-Acosta, E; Gutiérrez-Castorena, MC; Rubiños-Panta, JE; Alvarado-López, J. 2006. Caracterización del suelo y plantas de un sitio contaminado con hidrocarburos. Terra Latinoamericana 24(4):463-470.

Hernández-Acosta, E; Mondragón-Romero, E; Cristóbal-Acevedo, D; Rubiños-Panta, JE; Robledo-Santoyo, E. 2009. Vegetación, residuos de mina y elementos potencialmente tóxicos de un jal de Pachuca, Hidalgo, México. Revista Chapingo (Serie Ciencias Forestales y del Ambiente) 15(2):109-114.

Hernández-Valencia, I; Mager, D. 2003. Uso de Panicum máximum y Brachiaria brizantha para fitorremediar suelos contaminados con un crudo de petróleo liviano. Bioagro 15(3):149-155.
Hou, D; Ok, YS. 2019. Soil pollution - speed up global mapping. Nature 566(7745):455. DOI: https://doi.org/10.1038/d41586-019-00669-x.

Juárez-Cisneros, G; Sánchez-Yáñez, JM. 2014. Biorestauration of soil polluted by waste motor oil by biostimulation with vermicompost and phytoremediation with Sorghum vulgare inoculated by Bacillus cereus and Rhizobium etli. Journal of the Selva Andina Biosphere 2(1):1522.

Kantawanichkul, S; Kladprasert, S; Brix, H. 2009. Treatment of high-strength wastewater in tropical vertical flow constructed wetlands planted with Typha angustifolia and Cyperus involucratus. Ecological Engineering 35(2):238-247. DOI: http://doi.org/10.1016/j. ecoleng.2008.06.002.

Khalil-Gardezi, A; Barcelo-Quintal, ID; Cetina-Alcalá, VM; Bussy, AL; Pérez-Nieto, J; Borja-Salin, MA. 2006. Absorción de cobre y características de Leucaena leucocephala asociada con Glomus spp. y Rhizobium en suelo contaminado del Río Lerma, México. Terra Latinoamericana 24(3):347-354.

Labra-Cardón, D; Guerrero-Zúñiga, LA; Rodríguez-Tovar, AV; Montes-Villafán, S; PérezJiménez, S; Rodríguez-Dorantes, A. 2012. Respuesta de crecimiento y tolerancia a metales pesados de Cyperus elegans y Echinochloa polystachya inoculadas con una rizobacteria aislada de un suelo contaminado con hidrocarburos derivados del petróleo. Revista Internacional de Contaminación Ambiental 28(1):716.

Landeros-Márquez, O; Trejo-Calzada, R; Reveles-Hernández, M; Valdez-Cepeda, RD; Arreola-Ávila, JG; Pedroza-Sandoval, A; Ruíz-Torres, J. 2011. Potential use of huizache (Acacia farnesiana L. Will) in phytoremediation of lead-contaminated soils. Revista Chapingo (Serie Ciencias Forestales y del Ambiente) 17 n. ${ }^{\circ}$ esp. 1:11-20. DOI: http://doi.org/10.5154/r.rchscfa.2010.08.059.

López-Martínez, S; Gallegos-Martínez, ME; Pérez-Flores, LJ; Gutiérrez-Rojas, M. 2005. Mecanismos de fitorremediación de suelos con- 
taminados con moléculas orgánicas xenobióticas. Revista Internacional de Contaminación Ambiental 21(2):91-100.

Mager, D; Hernández-Valencia, I. 2013. Actividad microbiana durante la fitorremediación de un suelo contaminado con un crudo liviano. Revista de la Facultad de Agronomía (LUZ) (30):53-72.

Maldonado-Chávez, E; Rivera-Cruz, MC; Izquierdo-Reyes, F; Palma-López, DJ. 2010. Efectos de rizosfera, microorganismos y fertilización en la biorremediación y fitorremediación de suelos con petróleos crudo nuevo e intemperizado. Universidad y Ciencia 26(2):121-136.

Manios, T; Stentiford, EI; Millner, P. 2003. Removal of heavy metals from a metaliferous water solution by Typha latifolia plants and sewage sludge compost. Chemosphere 53(5):487-494. DOI: http://doi.org/10.1016/ S0045-6535(03)00537-X.

Marín-Velázquez, TD. 2016. Crecimiento de plantas de maíz (Zea mays) en un suelo contaminado con petróleo y remediado con extracto de cáscaras de naranja (Citrus sinensis). Enfoque UTE 7(3):1-13.

Marrugo-Negrete, J; Durango-Hernández, J; Pinedo-Hernández, J; Olivero-Verbel, J; Díez, S. 2015. Phytoremediation of mercury-contaminated soils by Jatropha curcas. Chemosphere 127:58-63. DOI: https://doi.org/10.1016/j.chemosphere.2014.12.073.

Martel-Valles, F; Benavides-Mendoza, A; Mendoza-Villarreal, R; Zermeño-González, A; Juárez-Maldonado, A. 2014. Agronomic use of produced water in tomato plants (Lycopersicon esculentum L.) under greenhouse conditions. Revista Internacional de Contaminación Ambiental 30(4):365-377.

Mendoza-Carranza, M; Sepúlveda-Lozada, A; Días-Ferreira, C; Violette, G. 2016. Distribution and bioconcentration of heavy metals in a tropical aquatic food web: a case study of a tropical estuarine lagoon in SE Mexico. Environmental Pollution 210:155-165. DOI: http:// doi.org/10.1016/j.envpol.2015.12.014.
Molnár, VE; Simon, E; Ninsawat, S; Tóthmérész, B; Szabó, S. 2020. Pollution assessment based on element concentration of tree leaves and topsoil in Ayutthaya province, Thailand. International Journal of Environmental Research Public Health 17(14):5165. DOI: https://doi. org/10.3390/ijerph17145165.

Muratova, AY; Dmitrieva, TV; Panchenko, LV; Turkovskaya, OV. 2008. Phytoremediation of oil-sludge-contaminated soil. International Journal of Phytoremediation 10(6):486-502. DOI: http://doi. org/10.1080/15226510802114920

Ochoa-Gaona, S; Pérez-Hernández, I; Frías-Hernández, JA; Jarquín-Sánchez, A; Méndez-Valencia, A. 2011. Estudio prospectivo de especies arbóreas promisorias para la fitorremediación de suelos contaminados por hidrocarburos. Tabasco, México, Colegio de la Frontera Sur. 145 p.

Olguín, EJ; Hernández, ME; Sánchez-Galván, G. 2007. Contaminación de manglares por hidrocarburos y estrategias de biorremediación, fitorremediación y restauración. Revista Internacional de Contaminación Ambiental 23(3):139-154.

Olmos-Márquez, MA; Alarcón-Herrera, MT; Martín-Domínguez, IR. 2012. Performance of Eleocharis macrostachya and its importance for arsenic retention in constructed wetlands. Environmental Science and Pollution Research (19):763-771. DOI: http://doi.org/10.1007/ s11356-011-0598-X.

Orchard, J; Doucette, J; Chard, K; Bugbee, B. 2000. Uptake of trichloroethylene by hybrid poplar trees grown hydroponically in flowthrough plant growth chambers. Environmental Toxicology Chemistry 19(4):895-903. DOI: https://doi.org/10.1002/etc.5620190416.

Orozco-Soto, M; Gutiérrez-Rojas, M; Peralta-Pérez, MR. 2014. Efecto de la composición de una mezcla de hidrocarburos en la respuesta fenotípica y la acumulación de azúcares por Bouteloua curtipendula Michx. Torr. en cultivos in vitro. Polibotánica (38):157-170.

Oyedeji, S; Olawale Raimi, I; Ifechukwude Odiwe, A. 2013. A comparative assessment of the 
crude oil-remediating potential of Cynodon dactylon and Eleusine indica. Environmental and Experimental Biology (11):145-150.

Pandey, VC; Pandey, DN; Singh, N. 2014. Sustainable phytoremediation based on naturally colonizing and economically valuable plants. Journal of Cleaner Production 86(30):1-3.DOI: http://doi.org/10.1016/j.jclepro.2014.08.030.

Peng, S; Zhou, Q; Cai, Z; Zhang, Z. 2009. Phytoremediation of petroleum contaminated soils by Mirabilis jalapa L. in a greenhouse plot experiment. Journal of Hazardous Materials 168(2):1490-1496. DOI: http://doi. org/10.1016/j.jhazmat.2009.03.036.

Peralta-Pérez, MR; Volke-Sepúlveda, TL. 2012. La defensa antioxidante en las plantas: una herramienta clave para la fitorremediación. Revista Mexicana de Ingeniería Química 11(1):7588.

Pérez-Hernández, I; Ochoa-Gaona, S; Adams-Schroeder, RH; Rivera-Cruz, MC; Geissen, V. 2013. Tolerance of four tropical tree species to heavy petroleum contamination. Water Air Soil Pollution 224(1637):1-13. DOI: http:// doi.org/10.1007/s11270-013-1637-7.

Pérez-López, ME. 2009. Selección de plantas acuáticas para establecer humedales en el estado de Durango. Tesis PhD. Chihuahua, México, CIMAV. 92 p.

Prieto-Méndez, J; González-Ramírez, CA; Román-Gutiérrez, AD; Prieto-García, F. 2009. Plant contamination and phytotoxicity due to heavy metals from soil and water. Tropical and Subtropical Agroecosystems 10(1):29-44.

Puga, S; Sosa, M; Mora, A; Pinedo, C; Jiménez, J. 2006. Concentraciones de As y $\mathrm{Zn}$ en vegetación nativa cercana a una presa de jales. Revista Internacional de Contaminación Ambiental 22(2):75-82.

Ramírez-Elías, MA. 2014. Microorganismos degradadores de hidrocarburos del petróleo aislados de la rizósfera de manglar en el estado de Campeche y su potencial en la biorremediación. Tesis PhD. Ciudad de México, México, Colegio de Postgraduados. 185 p.
Rivera-Cruz, MC; Maldonado-Chávez, E; Trujillo-Narcía, A. 2012. Effects of crude oil on the growth of Brachiaria mutica and Leucaena leucocephala and on soil and plant macronutrients. Tropical and Subtropical Agroecosystems 15 supl. 2:30-39.

Rivera-Cruz, MC; Trujillo-Narcía, A. 2004. Estudio de toxicidad vegetal en suelos con petróleos nuevo e intemperizados. Interciencia 29(7):369-376.

Rivera-Cruz, MC; Trujillo-Narcía, A; de la Miranda, CMA; Maldonado, CE. 2005. Evaluación toxicológica de suelos contaminados con petróleos nuevo e intemperizado mediante ensayos con leguminosas. Interciencia 30(6):326331.

Rivera-Ortiz, P; Rivera-Lárraga, JE; Andrade-Limas, EC; Heyer-Rodríguez, L; De La Garza-Requena, FR; Castro-Meza, BI. 2018. Bioestimulación y biorremediación de recortes de perforación contaminados con hidrocarburos. Revista Internacional de Contaminación Ambiental 34(2):249-262. DOI: http://doi. org/10.20937/RICA.2018.34.02.06.

Rivero-Casado, AN; Rodríguez-Vázquez, R; Montes-Horcasitas, MC; Pérez-Vargas, J; Gómez-Guzmán, O; Calva-Calva, G. 2010. Hidrocarburos aromáticos y fenilpropanoides presentes en la rizosfera de plantas de Cyperus laxus crecido en suelos contaminados con hidrocarburos. Tecnocultura (20):4-15.

Rodríguez-Eugenio, N; McLaughlin, M; Pennock, D. 2019. La contaminación del suelo: una realidad oculta. Roma, FAO. 92 p.

Rodríguez-Vázquez, R; Sánchez, S; Mena-Espino, X; Amezcua-Allieri, MA. 2016. Identification of the medicinal plant species with the potential for remediation of hydrocarbons contaminated soils. Acta Physiologiae Plantarum 38(1):23-34. DOI: http://doi.org/10.1007/ s11738-015-2036-Z.

Ruiz-Huerta, EA; Armienta-Hernández, MA. 2012. Acumulación de arsénico y metales pesados en maíz en suelos cercanos a jales o residuos mineros. Revista Internacional de Contaminación Ambiental 28(2):103-117. 
Salazar, MJ; Pignata, ML. 2014. Lead accumulation in plants grown in polluted soils. Screening of native species for phytoremediation. Journal of Geochemical Exploration 137:29-36. DOI: http://doi.org/10.1016/j.gexplo.2013.11.003.

Sangabriel, W; Ferrera-Cerrato, R; Trejo-Aguilar, D; Mendoza-López, MR; Cruz-Sánchez, JS; López-Ortiz, C; Delgadillo-Martínez, J; Alarcón, A. 2006. Tolerancia y capacidad de fitorremediación de combustóleo en el suelo por seis especies vegetales. Revista Internacional de Contaminación Ambiental 22(2):63-73.

SEMARNAT (Secretaría de Medio Ambiente y Recursos Naturales, México). 2010. Norma Oficial Mexicana de protección ambiental, especies nativas de México de flora y fauna silvestres, categorías de riesgo y especificaciones para su inclusión, exclusión o cambio y lista de especies en riesgo. NOM-059-SEMARNAT-2010. Ciudad de México, México. 30 dic. 2010.70 p.

Schnoor, JL; Licht, LA; McCutcheon, SC; Wolfe, NL; Carreira, LH. 1995. Phytoremediation of organic and nutrient contaminants. Environmental Science \& Technology 29(7):318-323. DOI: http://doi.org/10.1021/es00007a747.

The Plant List. 2013. The Plant List. Vers. 1.1 (en línea). Missouri, Estados Unidos de América. Consultado 1 ene. 2021. Disponible en http:// www.theplantlist.org/.

Valles-Aragón, MC; Alarcón-Herrera, MT. 2014. Retención de arsénico en humedales construidos con Eleocharis macrostachya y Schoenoplectus americanus. Revista Internacional de Contaminación Ambiental 30(2):143148.

Vázquez-Luna, D; Castelán-Estrada, M; Rivera-Cruz, MC; Ortiz-Ceballos, AI; Izquierdo, F. 2010. Crotalaria incana L. y Leucaena leucocephala Lam. (Leguminosae): especies indicadoras de toxicidad por hidrocarburos de petróleo en el suelo. Revista Internacional de Contaminación Ambiental 26(3):183-191.

Vázquez-Yanes, C; Batis-Muñoz, AI; Alcocer-Silva, MI; Gual-Díaz, M; Sánchez-Dirzo, C. 1999. Árboles y arbustos nativos potencial- mente valiosos para la restauración ecológica y la reforestación. México DF, México, UNAM. 13 p. Proyecto J-084-CONABIO.

Velasco-Trejo, JA; Volke-Sepúlveda, TL. 2003. El composteo: una alternativa tecnológica para la biorremediación de suelos en México. Gaceta Ecológica (66):41-53.

Zavala-Cruz, J; Gavi-Reyes, F; Adams-Schroeder, RH; Ferrera-Cerrato, R; Palma-López, DJ; Vaquera-Huerta, H; Domínguez-Ezquivel, JM. 2005. Derrames de petróleo en suelos y adaptación de pastos tropicales en el Activo Cinco Presidentes, Tabasco, México. Terra Latinoamericana 23(3):293-302.

Zezulka, Š; Klemš, M; Kummerová, M. 2014. Root and foliar uptake, translocation, and distribution of [14C] fluoranthene in pea plants (Pisum sativum). Environmental Toxicology and Chemistry 33(10):2308-2312. DOI: http:// doi.org/10.1002/etc. 2677 . 